\title{
A new Method for Efficient 3D Reconstruction of Outdoor Environments Using Mobile Robots
}

\author{
Jaime A. Pulido Fentanes \\ Fundación CARTIF \\ Parque Tecnológico de Boecillo, \\ Valladolid, \\ España, 47151 \\ jaipul@,cartif.es \\ Eduardo Zalama \\ Universidad de Valladolid, \\ ETSII, \\ Paseo del Cauce $\mathrm{S} / \mathrm{N}$, \\ Valladolid, España \\ ezalama@eis.uva.es
}

\author{
Raul Feliz Alonso \\ Fundación CARTIF \\ Parque Tecnológico de Boecillo, \\ Valladolid, \\ España, 47151 \\ raufel@cartif.es
}

\author{
Jaime Gómez García-Bermejo \\ Universidad de Valladolid, \\ ETSII, \\ Paseo del Cauce $\mathrm{S} / \mathrm{N}$, \\ Valladolid, España \\ jaigom@,cartif.es
}

\begin{abstract}
In this paper, a method for robotic exploration oriented to the automatic threedimensional reconstruction of outdoor scenes is presented. The proposed algorithm focuses on optimizing the exploration process by maximizing map quality, while reducing the amount of scans required to create a good quality 3D model of the environment. This is done by using expected information gain, expected model quality and trajectory cost estimation as criteria for view planning. The method has been tested with an all-terrain mobile robot which is also described in the paper. This robot is equipped with a Sick Lms 111 laser scanner attached to a spinning turret, which performs quick and complete allaround scans. Different experiments of autonomous 3D exploration show the suitable performance of the proposed exploration algorithm.
\end{abstract}

\section{Introduction}

In emergency situations, it is often necessary to obtain as much information as possible about a given area before planning and carrying out intervention actions. However, obtaining this information is not an easy task, especially in the case of large outdoor environments where access may be difficult and even unsafe for humans. In particular, 3D models represent a powerful and desirable description of the environment. However, up to now, most 3D scanning systems are of a stationary nature. This means that building a model of a large and complex environment requires several scans taken separately and from many different viewpoints. 
Unfortunately, setting up a typical stationary laser scanning system is a hard and time consuming job, since scanners are often heavy and scanning work requires many auxiliary elements (tripod, batteries, GPS antenna and computer) to be set up. Moreover, a human operator has to choose which views will be less occluded and will provide the highest amount of new information, and has to transport the whole system to the chosen location and set it up. The process is repeated until the operator decides that the scans taken are enough to cover the whole area with the desired quality. This decision is made using the operator's experience, and a close look at the resulting model is not taken until all the captured data is processed and aligned at a later step, often not in field. Then, if a portion of the environment has been missed, or the resulting model quality was not good enough, the scanning system has to be redeployed. This manual procedure is a big liability in emergency and disaster situations, where quickly obtained 3D models of the environment are vital to planning rescue operations. However, the main drawback for SSRR scenarios is the presence of the human operator in an environment which can be unsafe for his/her health (presence of toxic gases, fire, radioactivity and biological threats).

The use of mobile robots equipped with on-board 3D scanning systems emerges as a suitable alternative (Blaer \& Allen, 2007) (Nüchter, Lingemann, Hertzberg, \& Surmann, 2007), given that robots provide mobility, computing resources, localization systems and physical support to the scanning equipment. However, though the use of mobile robots can largely reduce the scanning process effort, the automation of the view selection process is still an open problem.

This issue has been addressed within the field of mobile robot exploration. However, most of the research effort has focused on the SLAM problem, so proposed techniques are designed to improve the extraction of relevant features along robot trajectories in order to provide robot localization and map information. Moreover, the main objective of these approaches is often to autonomously obtain indoor maps which help robots to self localise, and the mapping process is constrained to flat environments.

Outdoor environments have many characteristics that make the problem different from indoor scenarios. The environment is less structured: it is not limited by walls, the floor is not flat, there are arbitrary but relevant 3D elements (such as trees, stones and cars) and thus, mere 2D maps are not efficient for navigation. Moreover, 3D mapping becomes a complex problem due to the huge memory and computational resources required for data handling, besides other problems such as occlusions. Fortunately, the localization problem may be less severe in outdoor environments due to the availability of absolute localization sensors such as DGPS and compasses, which provide reasonably accurate robot localization.

In this context, the method presented in the present paper is focused on optimizing the exploration process by maximizing map quality, while reducing the amount of scans required to create a good quality 3D model of the environment. The goal is to obtain a robot that can efficiently build a model of its surrounding environment. The robot takes previous data into consideration in order to choose the best next locations to do a new scan, and computes the trajectory towards these locations. This methodology can be applied to most outdoor scenarios such as urban locations, monuments, archaeological sites and forests.

In the present paper, the robotic platform used to set up our method is first presented; then the algorithm for view planning is explained; and finally, results and conclusions are given. 


\section{Related Work}

3D mapping on the field of Safety, Security and Rescue Robotics (SSRR) has been thoroughly studied and proven to be very useful in these situations. For example in (Ohno, Tadokoro, Nagatani, Koyanagi, \& Yoshida, 2009) the authors developed a method to create a 3D map of an underground mall and subway station using odometry and an offline 3D scan matching method based on ICP and an additional gravitation constraint that was tested in a simulation of a terrorist attack. Another remarkable example is (Pathak, Birk, Vaskevicius, Pfingsthorn, Schwertfeger, \& Poppinga, 2010), where a method for 3D mapping based on pose-graph relaxation was developed and tested on different SSRR scenarios such as a collapsed car park and a flooding disaster. There are currently different projects oriented to creating methods for modelling outdoor structures. Most of these projects are intended for building large scale 3D models of cities and focus mainly on the data processing and fusion processes, rather than on the view planning problem. Some remarkable projects are the 3-D city model construction project at Berkeley (Früh \& Zakhor, 2003), the MIT City Scanning Project (Teller, 1997), the 4D Cities project at Georgia Tech (Dellaert, 2005) and Google Street View (Williams, 2010). In these projects, data processing is completed after the capture stage, so this phase is not automated.

Some other projects address 3D data processing during the capturing stage. Smarter Team (Jensen, Weingarten, Kolski, \& Siegwart, 2005) and (Pfaff, Triebel, Stachniss, Lamon, Burgard, \& Siegwart, 2007) is an example, where an effort to build an autonomous car is carried out in order to demonstrate fully autonomous navigation and 3D mapping in outdoor environments. In this case, a 3D data processing module is required for finding traversable areas in the environment. However, a human operator has to choose the waypoints that the robot has to follow, so the exploration of the environment is left to a human component.

(Nüchter, Lingemann, \& Hertzberg, 2006) applied the concept of SPLAM to outdoor 3D mapping and navigation. Under this concept, a mobile robot designed for outdoor scenarios would have to take a scan, align it with previous scans, extract drivable surfaces within the environment and then use this information to plan the next observation position. Unfortunately, the planning stage was not covered by this work.

On the other hand, several different exploration techniques have been proposed using $2 \mathrm{D}$ data within the field of robotic exploration for planar scenarios. Two main strategies are followed: strategies where a predefined behaviour is selected (such as wall following or moving to random positions) based on a priori information of the environment (Danner \& Kavraki, 2000), (Bourque \& Dudek, 1998), and strategies that predict which movement will most improve the robots' knowledge of the environment, using previously acquired information.

The first group of strategies lacks adaptability to unknown environments, where they tend to either leave unexplored areas or be highly inefficient. The second group of strategies has received more attention, since they use information from the environment to decide further actions; they are more adaptable to any kind of environment. In general, all these methods are known as next best view (nbv), given that their main focus consists in finding the next best observation position to make the exploration process efficient.

Common methods within $\boldsymbol{n} \boldsymbol{b} \boldsymbol{v}$ approaches are greedy methods (Tovey \& Koenig, 2003), where the robot moves to the closest location of interest; frontier based methods (Yamauchi, 1997), where candidate locations are generated on the frontier between the explored and unexplored areas of the environment; and information based strategies, which use evaluation functions 
where different criteria can be used to choose the next best position according to the selected criteria, for example travelling cost (Yamauchi, Schultz, Adams, \& Graves, 1998).

Among the information based methods, some use functions to predict the utility of a location. For example, the utility of a target location is defined as an expected information gain in (Newman, Bosse, \& Leonard, 2003), and travelling cost is combined with information gain in (Gonzalez-Baños \& Latombe, 2002), so that the next best view point is chosen to maximize coverage and reduce travelling distance.

Some strategies find interest areas within the map that are also used as criteria. For example, in (Grabowski, Khosla, \& Choset, 2003), relevant features within the map are included and used for evaluating the next best view, considering that seeing these regions will facilitate SLAM. These strategies are known as active SLAM strategies. However, all these methods were proposed for planar environments represented by a 2D map.

A different solution for outdoor 3D exploration is proposed in (Blaer \& Allen, 2009) . It works in two stages. In the first stage, a 2D floor map of the area that has to be scanned is used to find possible occlusions that a 3D scan would have from different floor points. The combination of viewpoints that requires the lowest number of scans to entirely cover the target area is then found with this process. Once a 3D scan has been done from each previously calculated viewpoint, a second view planning algorithm step is then used to cover all unpredicted occlusions on the model with as few scans as possible. However, in this latter work, a priori information of the environment is required; the method proposed here only uses information captured by the robotic system at the exploration time and, from that information, chooses the next best viewpoint for $3 \mathrm{D}$ reconstruction.

\section{Our 3D Reconstruction Process}

There are multiple methods for creating 3D models of outdoor environments, as discussed in the previous section. The focus of the present work is to analyse 3D data in order to improve the data acquisition process. We have developed a mobile robot equipped with a $3 \mathrm{D}$ scanner on a spinning turret that can take 3D scans from different viewpoints. Scans are taken at stationary robot locations, which eases data processing (for example, surface reconstruction through point triangulation) and allows higher $3 \mathrm{D}$ resolution to be reached.
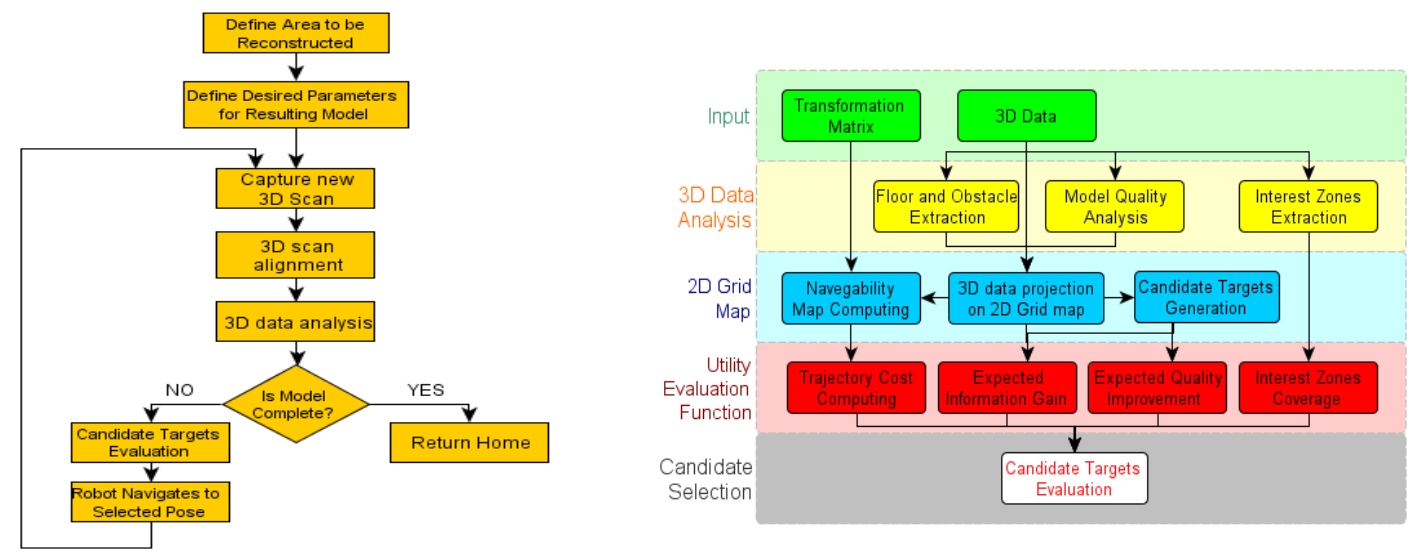

Figure 1- Block diagram of the 3D reconstruction process (left). Diagram of the exploration algorithm (right). 
Every time a new scan is acquired, the robot localization system is used to provide an initial pose hypothesis for the new data. Then the captured point cloud is aligned, using an ICP algorithm, with all previously captured point clouds to obtain an incremental model of the environment and a pose transformation matrix.

Figure 1 shows a block diagram of the proposed process. First, a 3D analysis process is carried out on this data to determine the robot navigation area and the model quality and to allow interest zone extraction. Then a 2D grid map is obtained from 3D data, which provides information about the environment navigability around the robot, and a group of random positions (candidate targets) is created. If the resulting model either does not comply with the specified quality requirements or does not cover the whole area, an exploration algorithm is used to evaluate which of those locations will be the next best position for taking a new scan.

The exploration algorithm (see Figure 1) is based on the multiple criteria $\boldsymbol{n} \boldsymbol{b} \boldsymbol{v}$ algorithm (Basilico \& Amigoni, 2009), where not only the distance and the expected information gain are considered for exploration, but also other critical information such as resulting model quality, view occlusion and navigation difficulty,

$$
u(t)=\frac{w_{A} A(t) \cdot w_{Q} Q(t) \cdot w_{O} O(t)}{w_{C} C(t)}
$$

In equation $1, u(t)$ is the utility evaluation function used by the algorithm, $t$ is the candidate position that is to be evaluated, $A(t)$ is a normalized value that represents expected information gain, $Q(t)$ is the improvement on model quality, $O(t)$ stands for the number and quality of interest zones covered from target $t, C(t)$ is a cost function that quantifies the difficulty of reaching each target, and $w_{A}, w_{Q}, w_{O}$ and $w_{C}$ are constant values that weight the influence of each criteria in the evaluation function.

All these criteria are chosen in order to obtain an environmental 3D model that fulfils model quality requirements while reducing the number of scans needed to cover the working area during movement, thus reducing the whole process time and the energy requirements. Once the new position has been chosen, the robot navigates to it using a reactive navigation system and repeats the process until the navigable area of the environment has been covered and the obtained model fulfils the quality requirements. All these steps will be described in detail in the following subsections.

\subsection{The All-terrain Robot and the 3D scanning system}

Our method has been designed to work on an all-terrain robot (see Figure 2) developed by us for three-dimensional outdoor measurement and reconstruction. The robot is a multi-purpose platform intended for the research of diverse applications in outdoor environments. It has a sixwheel differential traction system, with six independent BLDC motors (that provide robot mobility on irregular terrains), an electronic control system that controls motor traction, power and some low level sensors (temperature and sonars).

The robot can be used in either teleoperated, semi-autonomous or autonomous mode. To this end, it is equipped with an on-board PC system that controls other navigation sensors such as cameras, 2D laser, GPS and IMU, and gives support to any application software, and to a multichannel, long-range communication system for teleoperating or monitoring the robot in autonomous and semi-autonomous missions. 
A 3D scanning system (Figure 2) has also been designed within this work to address the 3D reconstruction task. It uses a 2D Sick LMS-111 laser system on a rotating platform that spins at a speed of 0.2 to $5 \mathrm{~Hz}$. Scan resolution depends on the spinning speed and the number of turns of the scanner. A typical scan has a pant-tilt resolution of $0.5^{\circ} \times 0.5^{\circ}$, and is taken in about 30 seconds.
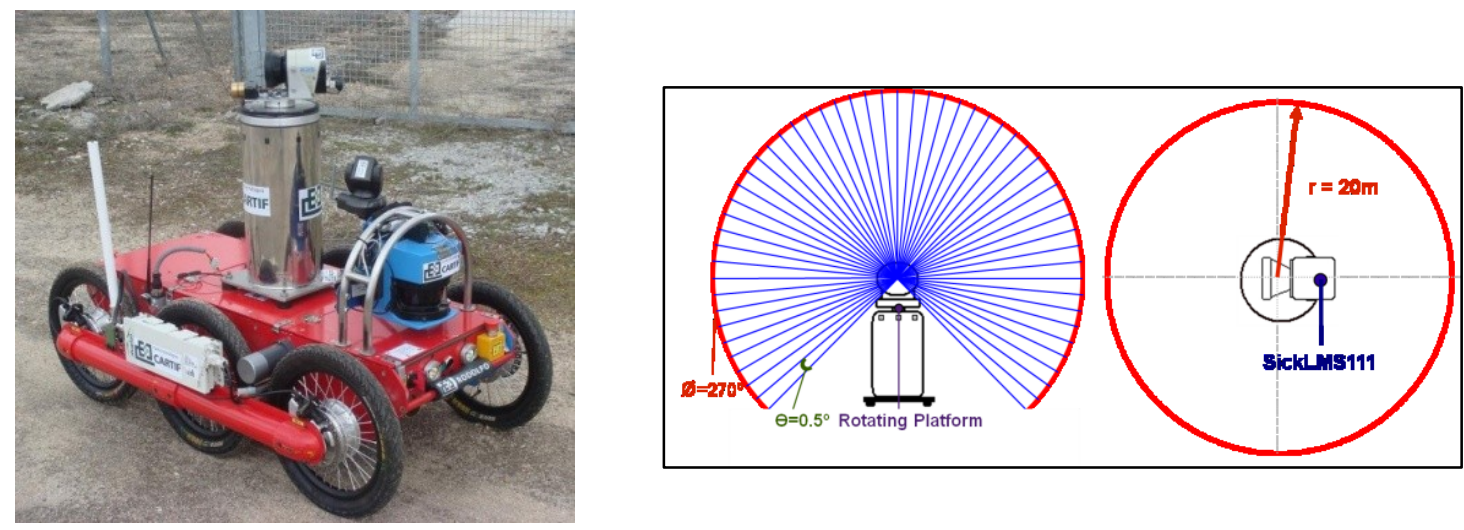

Figure 2 - All-terrain robot (left). Scheme of the 3D scanning system (right).

Moreover, since the development of an exploration algorithm requires extensive testing and the scanning of different environments from as many viewpoints as possible, simulation becomes a very desirable tool. For this reason, we have developed a specific 3D robotic environment simulator on which most tests can be run at a low cost. This tool has been developed in $\mathrm{C}++$ using ODE dynamic simulation libraries (Smith, 2007) for the simulation of physic variables and ray tracer. 3D data loading and processing has been implemented using Trimesh2 (Rusinkiewicz)libraries. Also "Inter Process Communication libraries" (Simmons, 2009) have been used for integrating the simulator with other control programmes such as the exploration algorithm proposed in the present paper. Thus, the transition from simulated environment and robot to real scenarios is quick and almost transparent to the user. The developed simulator has open software that can be downloaded at https://sourceforge.net/projects/simbot3d. Our method has been tested using both the mobile platform and the simulator, so comparative results can be seen in section 4 .

\subsubsection{D Reconstruction}

Once a new 3D model is captured, two different processes are used. The first one is the model triangulation; this process is necessary for most parts of the $3 \mathrm{D}$ analysis stage, since triangle information is used to evaluate model quality and extract safe navigation zones and obstacles. Also in this process, triangles with a bad geometrical shape are discarded and stored in a different list that is used for interest zones extraction.

The second process is the 6D SLAM that is used to create the transformation matrices for the system.

\section{Triangulation Process}

Taking advantage of the ordered way in which the system captures 3D data, triangles are created between neighboring points depending on their captured position; for example, the first point on 
a laser reading makes a triangle with the second point of that reading and the first point of the next reading. If a ray is over the maximal scanner reach the triangle is not created.

Once every triangle has been created every triangle is evaluated separately using the law of sines for relating the length of the sides of each triangle to the sines of its angles. If this relation is not within certain values $\left(\gamma_{\min }\right.$ and $\left.\gamma_{\max }\right)$ the triangle is discarded (see Figure 3).

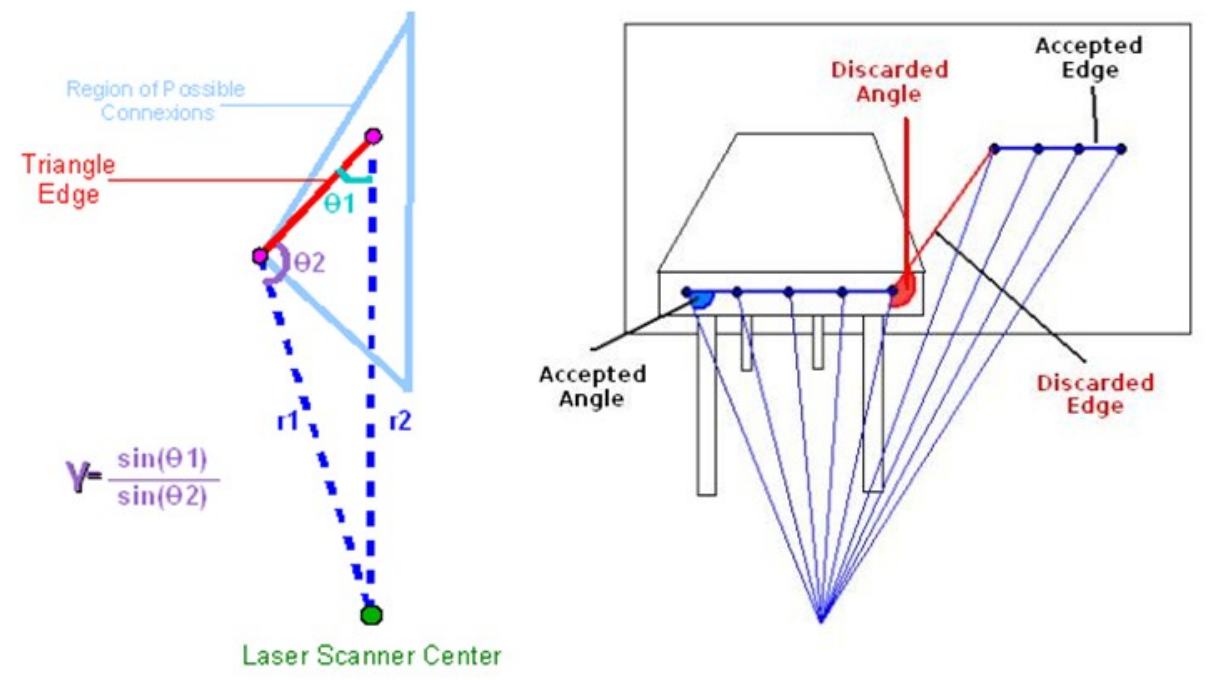

Figure 3 - Triangle Discard Methodology

Triangulation is done online with the 3D data capture process, the triangle discarding process takes less than 10 milliseconds after the data capture process is completed.

\section{D SLAM}

Following the 6D SLAM process presented by (Nüchter, Lingemann, Hertzberg, \& Surmann, 2007), we execute an ICP algorithm to align the last captured point cloud with its closest points clouds, giving it an initial pose estimation that is given by the robot's localization system. When a new loop closure is detected, a back propagation algorithm is run to realign the whole model. The model alignment process is the following:

1. Estimate robot position and orientation, using robot localization system.

2. Use these estimations to create an initial transformation matrix for the ICP alignment.

3. Align the scans using ICP.

4. If a loop closure is detected, back-propagate the error to previous registered scans.

Each alignment done with the ICP algorithm takes around 7 seconds. The back propagation algorithm runs only when a new loop closure is detected and needs 5 seconds per scan.

\subsection{D Data Analysis}

In this stage, the acquired 3D mesh is analyzed point by point to extract which points correspond to traversable surfaces and obstacles, to estimate model quality at each point and to extract interest zones from discarded triangles. This process is executed at every robot scan. 


\subsubsection{Extraction of safe navigation zones and obstacles}

3D data contains a large amount of information about the environment, and 3D points can correspond to obstacles, drivable surfaces (ground) or objects that the robot cannot reach (Nüchter, Lingemann, \& Hertzberg, 2006). The extraction of safe navigation areas is done by calculating the probability of each mesh point belonging to the ground (safe navigation zones) or to an obstacle. This data is useful to analyze navigability within the surrounding area.

If a point is at a reachable angle for the robot (i.e. the robot does not need to climb beyond its possibilities to reach this point), and its normal vector projection onto the world $\mathrm{Z}$ axis is large enough, this point has a high probability of belonging to a traversable zone from a local point of view.

However, neighboring points also have to be considered. For example, a point on an elevated plane may comply with local conditions, but its neighbor's probabilities could be much lower, so it should not be considered a safe navigation point. For this reason, the extraction of safe navigation areas is done in two steps. First, a probability from a local point of view $F_{p l}(p)$ is computed using (2), where $P z$ is the point height from the robot base, $d p$ is its distance to the scanner on the $\mathrm{XY}$ plane, $\mathrm{Nz}$ is its normal $\mathrm{Z}$ component on the global reference system and $\Theta$ (see Figure 4) is the maximum angle that the robot can climb.

$$
F_{p l}(p)=\left|N_{z}\right| \cdot\left(1-\frac{P_{z}-Z_{s c a n}}{d_{p} \sin (\theta)}\right)
$$
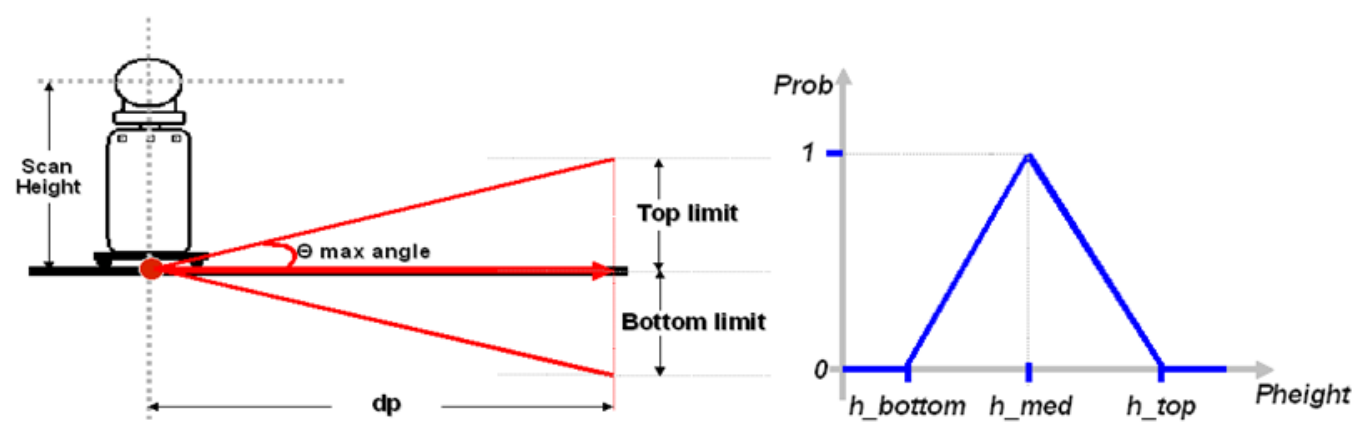

Figure 4 - Probabilities of belonging to a surface from point angle and distance

Then, a probability from a "global" point of view $F_{p g}(p)$ is computed as the average of each neighbouring point probability, $F_{p l}(p)$ (points sharing $3 \mathrm{D}$ mesh triangles with point $p$ ),

$$
F_{p g}(p)=\frac{1}{n} \sum_{i \in \gamma(p)} F_{p l}(i)
$$

Where $\gamma(p)$ is the set of neighbours of point $p$ and $n$ is the number of neighbours. Once $F_{p g}(p)$ has been computed, the final probability $F(p)$ for each point is obtained by weighting their corresponding probabilities with $w_{l}$ and $w_{g}$,

$$
F(p)=w_{l} F_{p l}(p)+w_{g} F_{p g}(p)
$$


A point can belong to an obstacle if it is at a reachable position for the robot and the plane to which it belongs is facing the robot (the dot product between the ray and the point normal vector is close to 1). Neighbours are also relevant since an obstacle point surrounded by floor points can be traversable. The probability of belonging to an obstacle is computed using a "local" probability function $B_{l}(p)(5)$ and a "global" probability function $B_{g}(p)(6)$ :

$$
\begin{aligned}
& B_{l}(p)=\left|N_{x y}\right|\left(\frac{\vec{P}}{|P|} \cdot \vec{N}\right) \\
& B_{g}(p)=\frac{1}{n} \sum_{i \in \gamma(p)} B_{l}(i)
\end{aligned}
$$

where $\left|N_{x y}\right|$ is the magnitude of the resulting vector addition of point normal components on the $\mathrm{X}$ and $\mathrm{Y}$ axis, $\overrightarrow{\boldsymbol{P}}$ is a vector from the 3D scanner to point $p$ and $\overrightarrow{\boldsymbol{N}}$ is its normal vector. In (6), the neighbours to point $p$ are used to find the global probability value. Then, the final probability $B(p)$ is obtained by weighting the $B_{l}(p)$ and $B_{g}(p)$ relevance with $w_{b l}$ and $w_{b g}$,

$$
B(p)=w_{b l} B_{l}(p)+w_{b g} B_{g}(p)
$$

Ground and obstacle probability values are used to create a navigation map that can be used to find trajectories and calculate route difficulty, as will be explained in section 3.3. All the results of this process will be shown in section 4 .

\subsubsection{Model quality analysis}

Model quality has to be analyzed point by point because it is not a homogeneous characteristic and is affected by various factors within one scan. In the analysis process, each point $p$ gets a score $\boldsymbol{A P}(\boldsymbol{p})$ (between 0 and 1) where 0 corresponds to bad quality and 1 to the desired quality or better. In this case Model quality is measured using point area and ray incidence angle as criteria. These criteria were chosen because they can be calculated in a quick and simple way, and they give a good idea of the resulting quality of the mesh, due to the fact that they provide information on the resolution of the scan at each point and how trustworthy the information provided by each point is. This score is calculated using the following equations:

$$
A A(p)=\left\{\begin{aligned}
\left(\frac{A_{\max }}{P_{A r}}\right), & P_{A r}>A_{\max } \\
1, & P_{A r} \leq A_{\max }
\end{aligned}\right.
$$

where $A A(p)$ is a function that compares the current area per point against the maximum desired area for point $p$, where $P_{A r}$ is a point $p$ area which is easily computed by adding one third of the area of each triangle that point $p$ belongs to. $A_{\max }$ is the maximum desired point area and is a parameter given to the algorithm that depends on desired scan resolution and maximum distance to an object at scanning.

$$
A I_{p}(p)=\left(\frac{\vec{P}}{|P|} \cdot \vec{N}\right)
$$


$A I_{p}(p)$ is a quality factor that depends on the ray incidence angle for the point's plane, $\overrightarrow{\boldsymbol{P}}$ is a vector from the $3 \mathrm{D}$ scanner to point $p$ and $\overrightarrow{\boldsymbol{N}}$ is its normal vector.

$$
A P(p)=\frac{\sqrt{\left[\omega_{a} A A(p)\right]^{2}+\left[\omega_{n} A I_{p}(p)\right]^{2}}}{\sqrt{\left(\omega_{a}^{2}+\omega_{n}^{2}\right)}}
$$

$A P(p)$ is the final score for each point, where $\omega_{a}$ and $\omega_{n}$ weight the relevance of the area against the ray incidence angle. Usually, $\omega_{a}$ is higher than $\omega_{n}$, since point area is the most relevant factor, and only really low scores on the ray incidence quality should affect the overall score. Figure 5 shows an example of the quality analysis process on a simple simulated environment using both criteria.
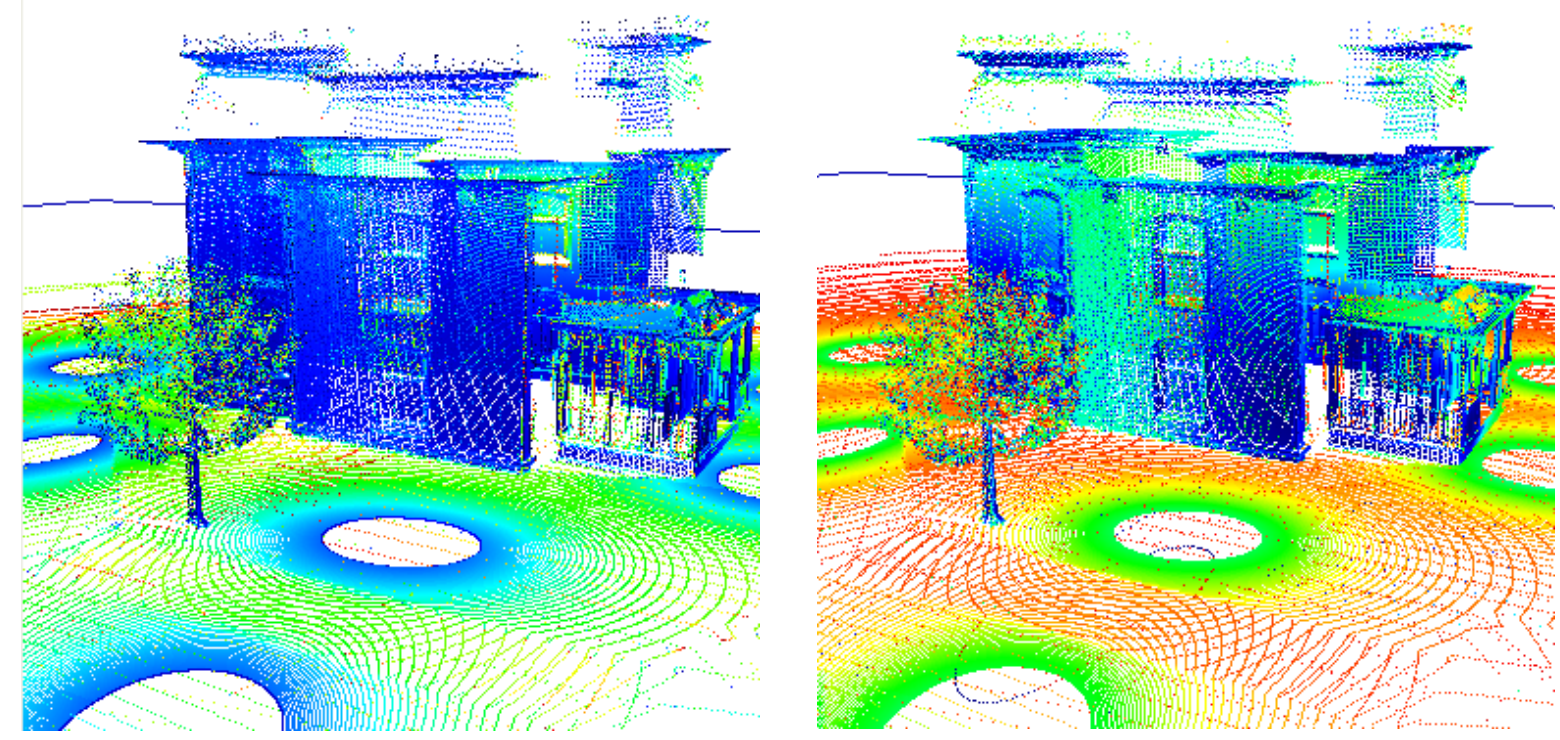

Figure 5 - quality score using point area (left); quality score by ray incidence angle (right). Colder colours indicate higher quality scores.

\subsection{Candidate Target Evaluation}

Candidate evaluation using 3D data can be a really time consuming process that also needs a great amount of resources. For this reason, a 2D information grid is used in this work to keep time and resources low without losing information from 3D data. Each cell in this grid stores all the information from an area of the environment, so processing information becomes much simpler. All the information of a mesh is projected onto this grid every time a new scan is taken. The resulting representation is useful for many different tasks, such as computing navigation maps by analyzing the amount of points that have a high probability of belonging to obstacles, or traversable surfaces in a cell.

\subsubsection{Extraction of interest zones}

Interest zones are extracted from models' discarded triangles, which are usually occluded planes. A vector pointing to the centre of each discarded triangle is created. These vectors are stored on a 
list, and are used to measure how well interest zones will be scanned from each candidate position. The resulting list is projected onto a $2 \mathrm{D}$ representation where information on which occlusion planes are covered from each evaluated target $t$, can be extracted using

$$
O(p)=\sum_{j \epsilon \delta} \sum_{i \epsilon \beta_{j}}\left|\overrightarrow{r_{e}} \cdot \overrightarrow{V_{l}}\right|
$$

where $\delta$ is the set of visible cells from target $t, \beta$ is the set of interest zones stored for each cell $j$, $\overrightarrow{r_{e}}$ is a vector from the 3D scanner to the point that marks the centre of an interest zone and $\vec{V}_{l}$ is the vector that is normal to the occlusion plane. Interest zones extracted from a simple simulated environment are shown in Figure 6.

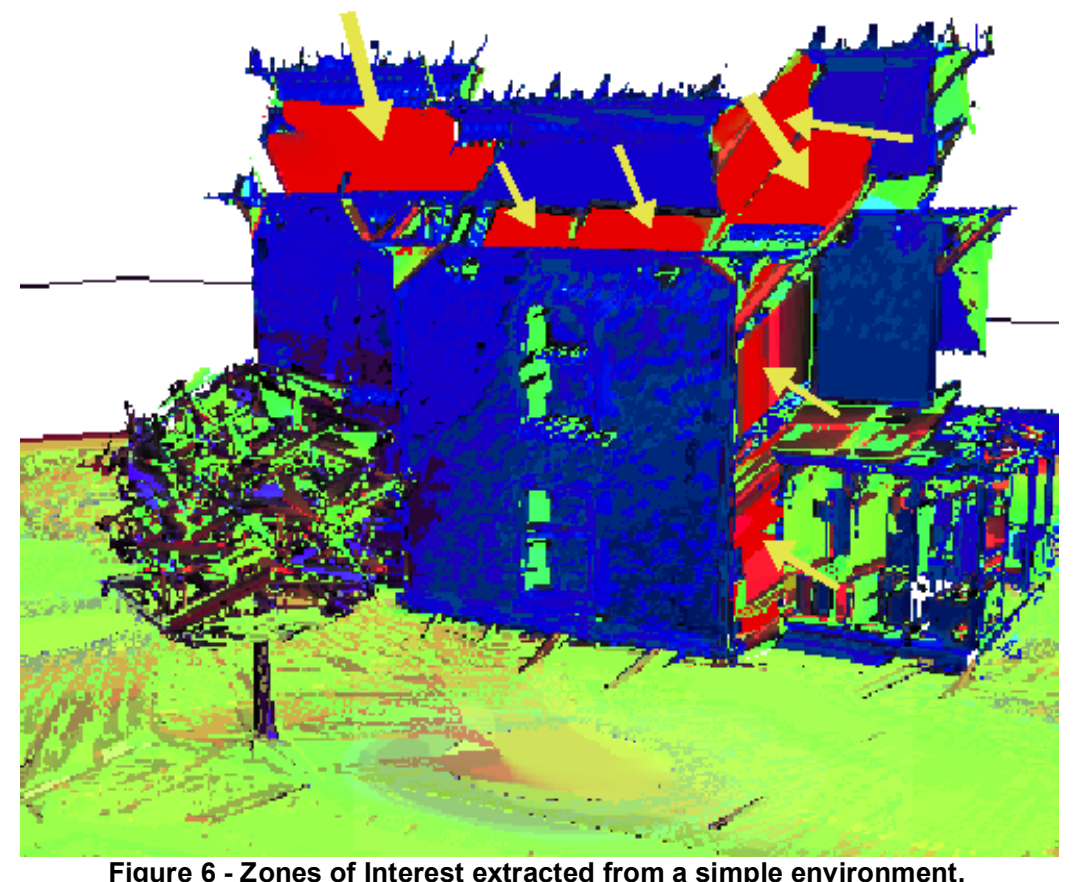

\subsubsection{D navigation map}

This representation is based on the navigability concept (Hertzberg, Lingemann, Christopher, Nüchter, \& Stiene, 2008). It is a map very similar in appearance to the occupancy grid maps used for 2D environments. However, cells in occupancy maps contain the probability of a cell being occupied by an object, while cells in the navigation map contain the probability of a cell being traversable by the robot.

Each time a new point with a high probability of belonging to a traversable surface is added to a cell, the probability of this cell being traversable increases. Otherwise, if an obstacle point is added, then this probability decreases. Navigability per cell $C_{n c}(c)$ ranges from 0 to 1 , where 1 corresponds to a completely traversable cell. This map is computed using all points that have a probability of belonging to a traversable surface over a given $\varepsilon_{f}$ value, or a probability of belonging to an obstacle over a $\varepsilon_{o}$ value.

$$
r_{f o}=\frac{n_{p f}}{n_{p f}+n_{p o}}
$$




$$
\begin{gathered}
F_{c}(c)=\sum_{i \in \varphi} F(i) \\
B_{c}(c)=\sum_{i \in \alpha} B(i)(13) \\
C_{n c}(c)=0.5+\left(r_{f o} \frac{n_{p f} F_{c}(c)}{2}\right)-\left(\left(1-r_{f o}\right) \frac{n_{p o} B_{c}(c)}{2}\right)
\end{gathered}
$$

In this expression, $\varphi$ is the set of points on cell $c$ with $F(p) \geq \varepsilon_{f}, n_{p f}$ is the number of points in the set $\varphi, \alpha$ is the set of cell points with $B(p) \geq \varepsilon_{o}$ and $n_{p o}$ is the number of points in the group $\alpha$. Candidate targets are generated in cells where $C_{n c}(c)>0.6$, so every evaluated target is reachable. Targets are distributed uniformly around the robot's position; so many different viewpoints are evaluated.

\subsubsection{Expected information gain}

The amount of new information that can be acquired from each evaluated candidate is computed upon the number of points and the minimum and maximum point heights per cell. This data is used to compute how many new cells will be scanned from a given target and which cells will be occluded by other ones.

In concrete, the first relevant value is the area covered from each candidate target $A_{n}(t)$, which is obtained using:

$$
A_{n}(t)=\frac{A_{c} C_{s e}}{\pi r_{e}^{2}}
$$

where $A c$ is the area represented by each cell and $C_{s e}$ is the number of unexplored cells within the scanner range. Computation is refined by subtracting the area of occluded cells $A_{o}(t)$ from the unexplored area that could be covered from a given candidate target.

Occluded cells are computed using a height map that is created using per cell min and max point heights; as well as using the information of the closest explored cell in the robot direction for unexplored cells.

Three points $m_{k}$ are generated at each cell: one point at the minimum height of 3D points corresponding to this cell, another one at the maximum height that the scanner could reach on that cell from the evaluated target, and a third one at the middle of the said points. Then, rays are traced from the scanner position at the evaluated target $t$, to these three points, and lines that cross a cell under its maximum height $n_{i l}$ are counted up. The occluded area is then computed using

$$
A_{o}(t)=\frac{n_{i l} A_{c}}{3}
$$

Finally, the expected information gain, $A(t)$, is obtained upon (16) and (17) using

$$
A(t)=A_{n}(t)-A_{o}(t)
$$

\subsubsection{Expected model quality improvement}

Model quality gain is the difference between quality information stored in the 2D information map and the quality information after a scan is taken from the evaluated target $t$.

Expected quality is calculated using two terms. The first one is the expected per cell point area $E Q_{A P}$, which is computed using the distance from the candidate target to each cell $r_{e}$, the maximum desired point area $A_{\max }$ and Pan-Tilt resolutions $\operatorname{res}_{p}$ and res $_{t}$. 


$$
\begin{gathered}
P A_{c}(c)=r_{e}^{2}\left(\sin \left(r e s_{p}\right) \sin \left(\text { res }_{t}\right)\right) \\
E Q_{A P}(c)=\left\{\begin{aligned}
\frac{A_{\max }}{P A_{c}(c)}, & P A_{c}(c) \geq A_{\max } \\
1, & P A_{c}(c)<A_{\max }
\end{aligned}\right.
\end{gathered}
$$

The second term corresponds to the quality improvement computed from the laser incidence angle. A new ray incidence quality for each point on the cells within the scanner reach range from the evaluated target, $E I_{C}(c)$, is computed using:

$$
E I_{c}(c)=\frac{1}{n p_{c}} \sum_{k \in \varphi}\left(\frac{\overrightarrow{R_{k}}}{\left|R_{k}\right|} \cdot \overrightarrow{N_{k}}\right)
$$

where $\varphi$ is the set of points stored in each cell, $n_{p c}$ is the number of points in each cell, $\overrightarrow{\boldsymbol{R}}$ is a vector from the evaluated target to each cell point and $\vec{N}$ is a unit vector normal direction to each cell point. Expected quality gain $Q(t)$ is obtained using:

$$
Q(t)=\sum_{c \in \sigma}\left(\frac{\sqrt{\left[\omega_{a} E Q_{A P}(c)\right]^{2}+\left[\omega_{n} E I_{c}(c)\right]^{2}}}{\sqrt{\left(\omega_{a}^{2}+\omega_{n}^{2}\right)}}-\frac{1}{n p_{c}} \sum_{p \in \varphi} A P(p)\right)
$$

where $\sigma$ is the set of cells within the range of the scanner, $A P(p)$ is the quality per point mark computed in section 3.2.2, and $\omega_{a}$ and $\omega_{n}$ are the values introduced in that section.

\subsubsection{Trajectory cost evaluation}

Trajectory cost evaluation is done by adding the difficulty of crossing each cell on the trajectory. This difficulty depends on the slope of each cell, the difference between the entry and the exit angle for each cell, the navigability $C_{n c}(c)$ value computed in section 3.3 .2 and the distance between cells $d_{e c}$. Trajectory cost is computed using:

$$
\left.C_{c}(c)=d_{e c} \frac{f_{g a}\left(\frac{h \min _{c i}-h \min }{c i+1}\right)}{\sin ^{-1}\left(\theta_{\max }\right)}\right)
$$

where $f_{g a}$ is a difficulty scale factor that depends on the trajectory curvature (see Figure 7), $c i$ is the current cell in the trajectory and $\Theta_{\max }$ is the maximum slope that the robot can climb.

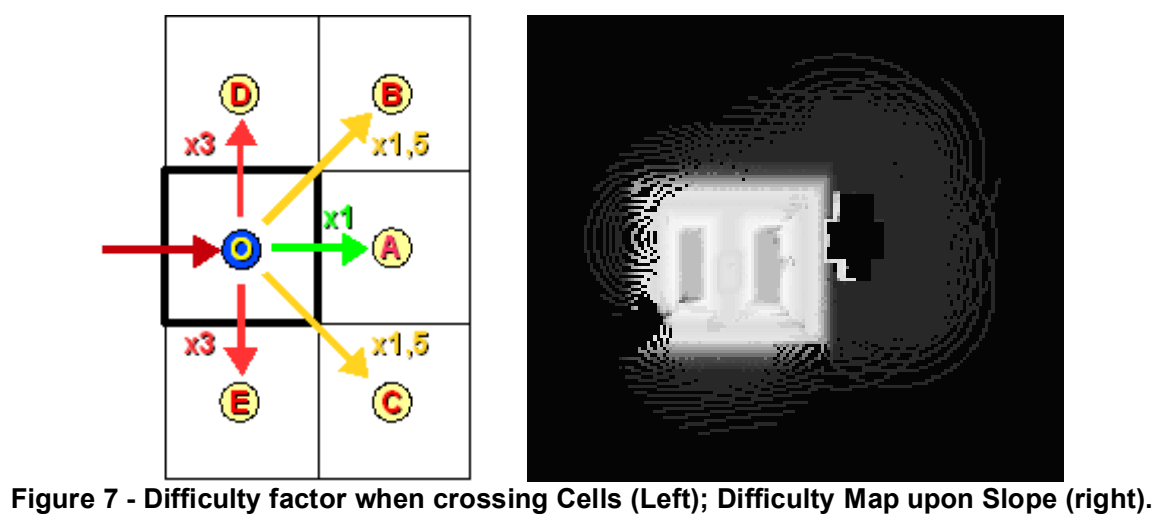




\section{Experimental Results}

In this section, results obtained with both real and simulated environments will be shown and discussed. First, some general considerations are made about parameter settings, and the parameter values chosen for the experiments are given. Then, results from different stages of the process are given, as well as the final models obtained after the reconstruction is completed. Three simulated environments and two real environments will be analysed.

All experiments were stopped when over $90 \%$ of the exploration area was covered or when the winning target score was less than 0.001 , which in simple words means that the effort of getting to a target is 1000 times greater than the utility.

\subsection{Parameter Setting}

Table I summarizes which stage is affected by each parameter, their value ranges and the effect that increasing or decreasing these values has on the algorithm. In all the experiments below (on both real and simulated environments), the parameter values shown in the last column of Table I were used.

Table I

Parameter values used in simulated and real environments

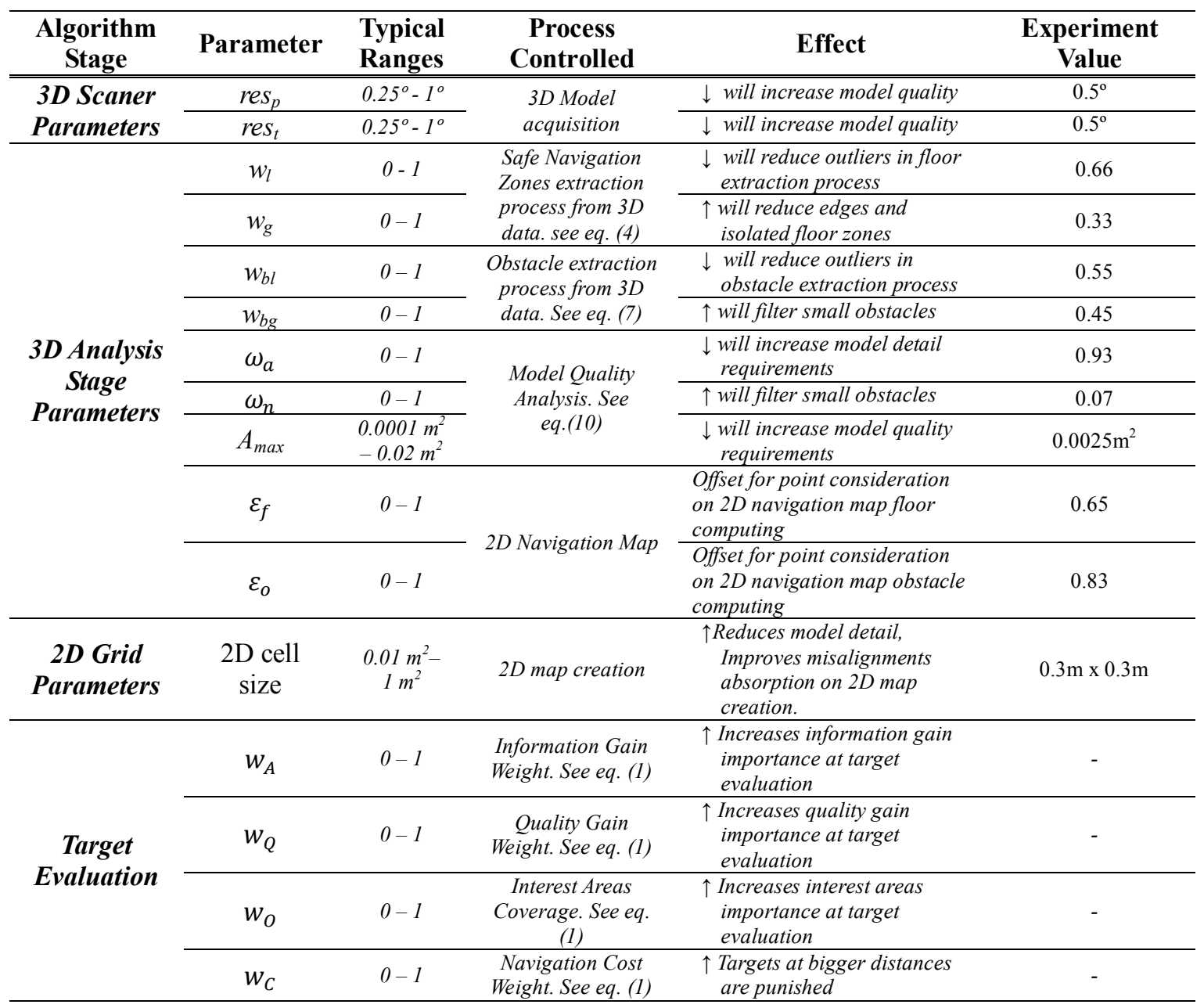


Please note that the parameters in equation 1 are not presented in the last column, given that they have been tuned separately for each experiment, in order to test specifically how they affect the behaviour of the algorithm.

\section{Other Considerations for Parameter Tuning}

For floor extraction, a higher $\boldsymbol{w}_{l}$ value leads to better results. Moreover, $w_{l}$ and $w_{g}$ should be set to 1 , unless most points want to be considered as floor ones. In this case, these values could be larger than 1 . On the contrary, values under 1 could be suitable when most points in the floor should not be considered. Once the desired floor extraction behaviour has been obtained, the values of these parameters can remain constant during the whole exploration process. For the 2D Navigation map, $\varepsilon_{\mathrm{f}}$ and $\varepsilon_{\mathrm{o}}$ values behave as offsets in order to consider only those points over a specific score when creating the map.

Model quality analysis is done in equation (10). The $A_{\max }$ value involved in this equation (through $A A(p)$ parameter) is the desired 3D model resolution. A suitable value for $A_{\max }$ can be calculated by providing the ideal distance to objects $\left(d_{o b j}\right)$ and the angular resolution of scan (res $p$ and $\mathrm{res}_{t}$ ), by means of:

$$
A_{\max }=d_{o b j}^{2}\left(\sin \left(r e s_{p}\right) * \sin \left(r e s_{t}\right)\right)
$$

The most relevant parameters are those for target evaluation (equation (1)). These parameters affect the behaviour of the algorithm. In concrete, $\boldsymbol{w}_{A}, \boldsymbol{w}_{\boldsymbol{Q}}$ and $\boldsymbol{w}_{\boldsymbol{O}}$ can be given values between 0 and 1 , proportionally to the desired influence of the corresponding term. However, a value of $w_{O}$ over 0.25 is not recommended since it might lead the system to behave unexpectedly.

Finally, the $w_{c}$ parameter can be computed as the inverse of the minimum desired distance to the next target on plane environments (e.g. 0.2 value for targets at a distance of at least 5 meters).

\subsection{Simulated Environments}

We have tested our method in three different simulated environments. The first one is a simple environment with only one building and a tree, placed on an irregular hill-shaped terrain. This environment has been used for evaluating how the different parameter values affect the behaviour of the algorithm, and how slopes may affect the navigability analysis and the trajectory cost estimation. The other two environments are more complex. The second one is a structured urban environment, intended for testing how the robot would move in an environment where navigation complexity is not high and the system can freely move along many different trajectories. The last simulated environment is the most complex. It is a cluttered outdoor scenario where only some paths are safe for the robot, so navigation complexity is high. The objective was to see if the algorithm can choose targets that are safe, and can efficiently manage cluttered environments. 


\subsubsection{Simple Environment}
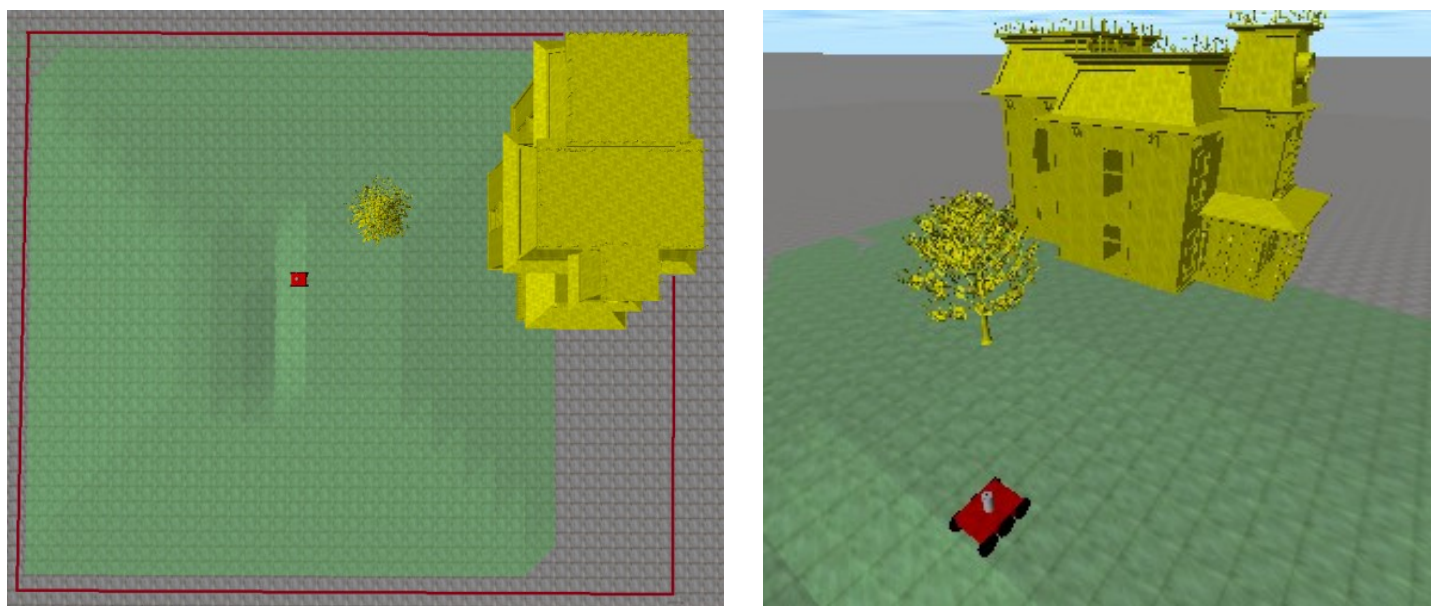

Figure 8 - Screenshot of the simple environment. The exploration area is squared in red.

Three experiments were carried out in this environment (Figure 8), using three different value choices for parameters in (1) in order to achieve different objectives. In the first experiment, the parameters were set in a balanced way; in the second experiment, information gain was given more importance than the other parameters, and in the third experiment, model quality was the dominant criterion. The chosen parameters can be seen in Table II.

Table II

Parameters Used In The Experiments

\begin{tabular}{ccccc}
\hline & $\mathbf{w}_{\mathbf{A}}$ & $\mathbf{w}_{\mathbf{Q}}$ & $\mathbf{w}_{\mathbf{O}}$ & $\mathbf{w}_{\mathbf{C}}$ \\
\hline \hline Experim. I & 0.43 & 0.35 & 0.22 & 0.09 \\
Experim. II & 0.65 & 0.25 & 0.10 & 0.09 \\
Experim. III & 0.36 & 0.5 & 0.14 & 0.09 \\
\hline
\end{tabular}

The $w_{c}$ parameter was not modified, so trajectories are determined only by the desired model criteria. Resulting trajectories can be seen in Figure 9.
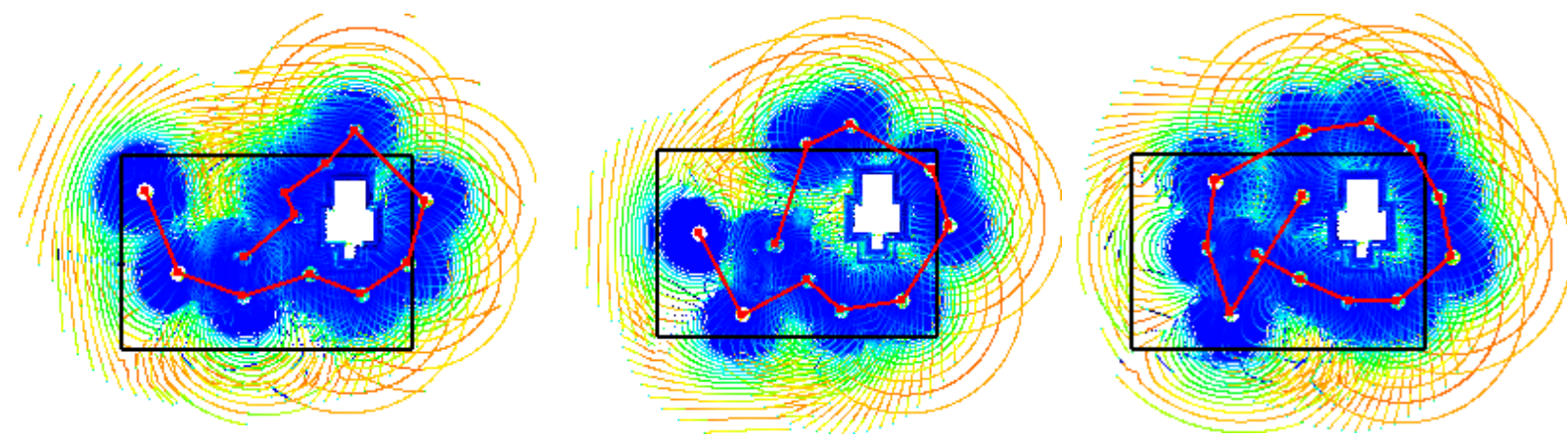

Figure 9 - Resulting trajectories for Experiment I (left); Experiment II (centre); Experiment III (right). Colder Colours Represent Better Quality

A fourth experiment with an implementation of a greedy mapping algorithm was carried out for comparison. In this experiment the system goes to the position where the estimation of information gain is the highest. The resulting trajectory is shown in Figure 10. 


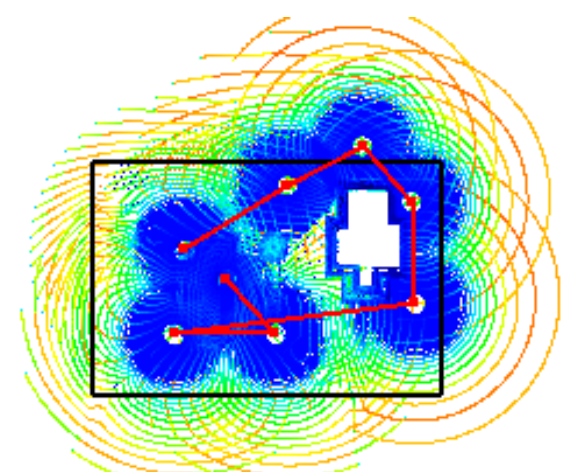

Figure 10 - Resulting trajectory for greedy mapping algorithm. Colder Colours Represent Better Quality

Table III shows the result for each experiment in terms of travelled distance, the number of scans, the amount of cells explored within the given area and a quality score computed by the averaging of the per-point quality score on each environment cell.

Table III

Results Obtained In Experiments

\begin{tabular}{ccccc}
\hline & $\begin{array}{c}\text { Travel } \\
\text { Distance }\end{array}$ & $\begin{array}{c}\text { Quality } \\
\text { Score }\end{array}$ & Coverage & Scans \\
\hline \hline Exp. I & $106 \mathrm{~m}$ & 0.6846 & $92.5 \%$ & 12 \\
Exp. II & $107 \mathrm{~m}$ & 0.6904 & $94.1 \%$ & 10 \\
Exp. III & $128 \mathrm{~m}$ & 0.7503 & $93.6 \%$ & 13 \\
Greedy & $116 \mathrm{~m}$ & 0.5013 & $91.4 \%$ & 8 \\
\hline
\end{tabular}

From these results, it can be seen that experiment II proved to be the most efficient solution, since it covered the entire area using only ten scans, whilst travelling only 1 meter more than the shortest trajectory. Experiment I led to a very similar model, but extra scans were required. Finally, experiment III required 13 scans to cover the whole area; however it led to a very high quality model. In comparison with the greedy mapping algorithm, the proposed algorithm may require more scans to achieve the same level of coverage. However, it provides a much higher quality score. The difference in terms of travelled distance and coverage is not noticeable, but the trajectory is inefficient for the greedy method since it requires the robot to execute rougher turns to reach the targets. Finally, there is an important difference in the quality distribution, as it is much more even in the experiments carried out with the proposed algorithm. Figure 11shows the simulated environment and a screenshot of the reconstructed model.

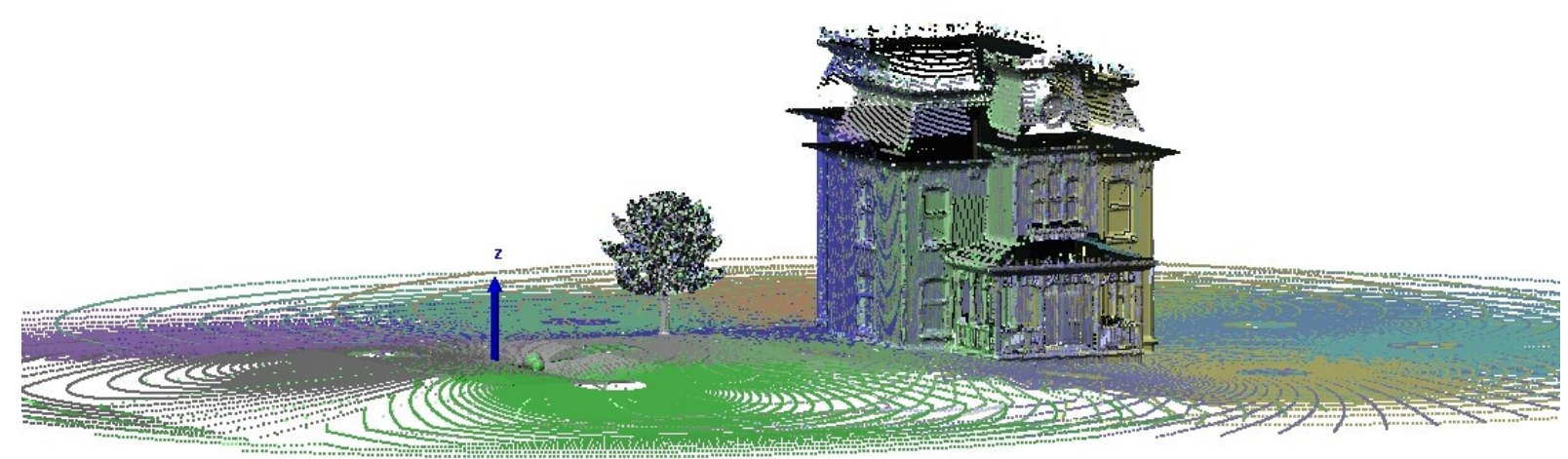

Figure 11 - Screenshot of the reconstructed model. 


\subsubsection{Structured Environment}

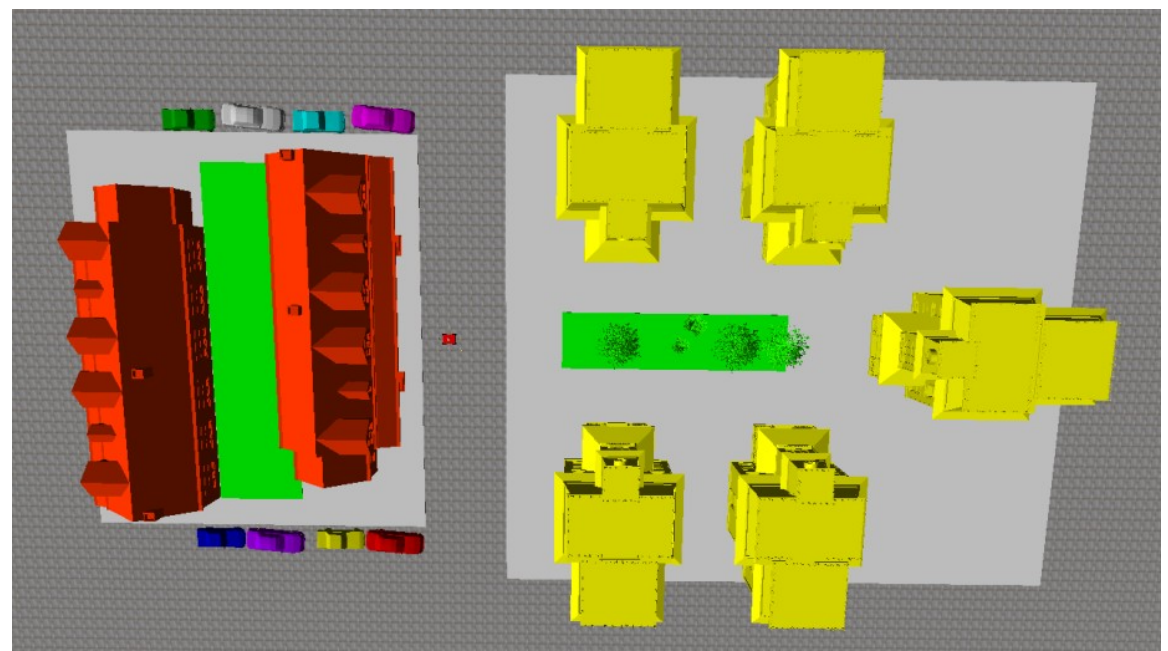

Figure 12 - Simulated structured environment

Figure 12 shows the simulated structured environment. Two experiments were carried out on this environment following two different strategies. In the first experiment, interest zones and map quality were chosen as the dominating criteria; in the second experiment, information gain was the dominating criterion and navigation cost weight was also slightly lower. Table IV shows the selected parameter values.

Table IV

Parameters Used In The Experiments

\begin{tabular}{ccccc}
\hline & $\mathbf{w}_{\mathbf{A}}$ & $\mathbf{w}_{\mathbf{Q}}$ & $\mathbf{w}_{\mathbf{O}}$ & $\mathbf{w}_{\mathbf{C}}$ \\
\hline \hline Exp. I & 0.2 & 0.55 & 0.25 & 0.09 \\
Exp. II & 0.65 & 0.25 & 0.10 & 0.09 \\
\hline
\end{tabular}

The resulting trajectories for both experiments are show in Figure 13.
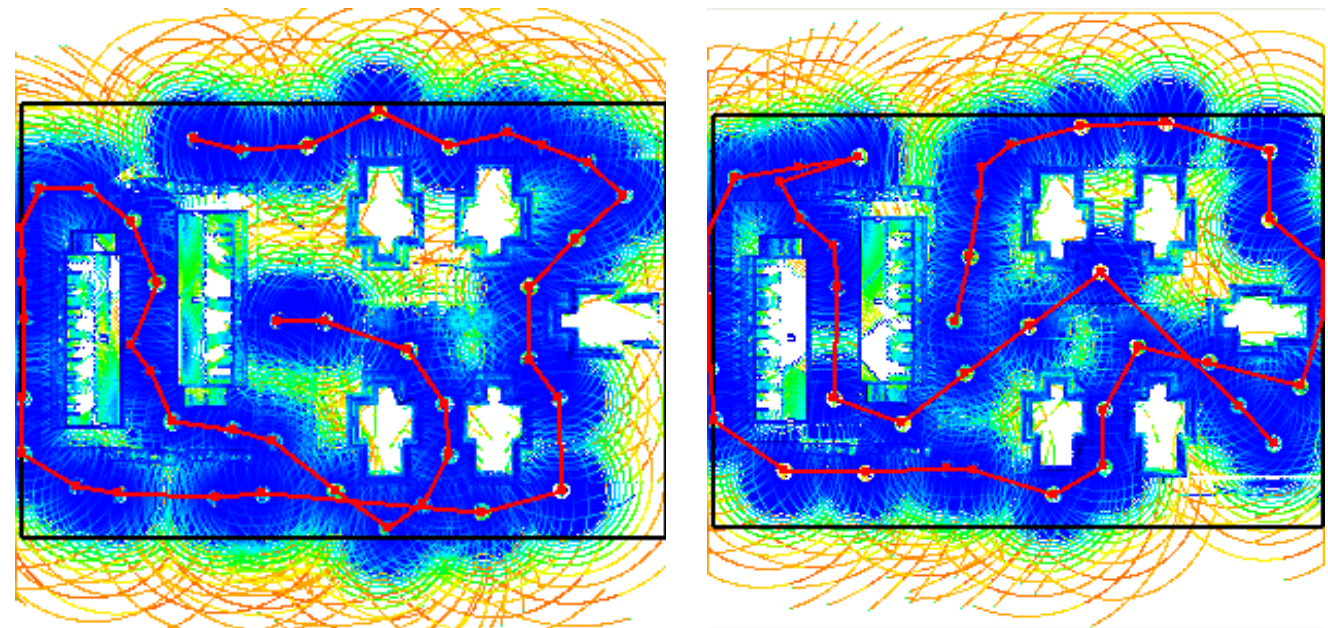

Figure 13 - Resulting Trajectories for Experiment I (left) and Experiment II (right). Colder Colours Represent Better Quality 
Table V shows the result for both experiments in terms of travelled distance, the number of scans done in each experiment, the amount of cells explored within the given area and the quality score defined in the previous subsection.

Table V

Results Obtained In Experiments

\begin{tabular}{ccccc}
\hline & $\begin{array}{c}\text { Travel } \\
\text { Distance }\end{array}$ & $\begin{array}{c}\text { Quality } \\
\text { Score }\end{array}$ & Coverage & Scans \\
\hline \hline Exp. I & $352 \mathrm{~m}$ & 0.6813 & $95 \%$ & 43 \\
Exp. II & $397 \mathrm{~m}$ & 0.726 & $94.8 \%$ & 39 \\
\hline
\end{tabular}

From these results, it can be seen that the algorithm can be used efficiently on structured environments. Also, it can be noted that both strategies are useful and have similar results, the difference in terms of coverage and quality scores is very small, and the longest trajectory travelled by experiment II is compensated by a smaller number of scans. It is important to mention that, due to the difference in target evaluation weight, both trajectories started out in very different ways. However, both experiments had very similar results, which may be because of the highly structured environment designed for the test, since interest zones and quality gain have very similar scores on targets all over the map, so information gain became the dominant criterion in both cases. Experiments in a cluttered environment will show that this happens only on structured scenarios.

Figure 14 shows the 2D navigation map and the final reconstructed model for experiment II.
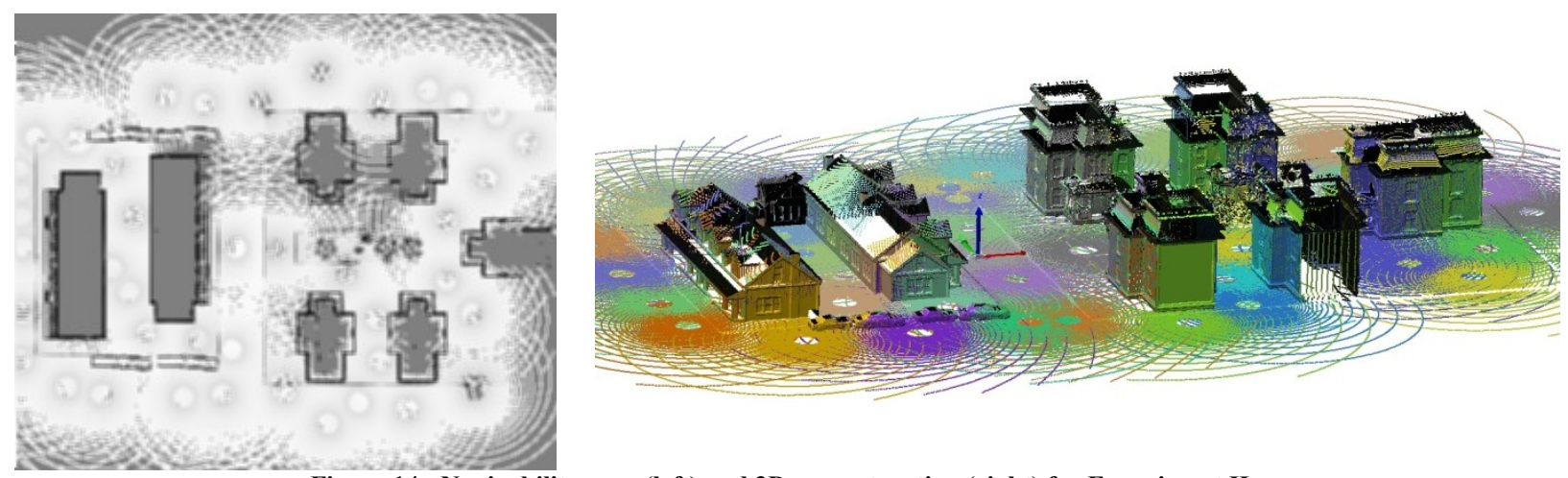

Figure 14 - Navigability map (left) and 3D reconstruction (right) for Experiment II

\subsubsection{Cluttered Environment}

A cluttered environment can be seen in Figure 15. It can be seen that the terrain has few safe paths and navigation complexity is very high. The environment is designed in such a way that the robot has to cross under and over bridges (in gray, in the figure) so that the ability of the system to manage gaps and overhanging structures can be tested.

The starting position is on the upper right corner of the environment, and the robot has to find suitable paths to cover the whole environment. The objective of this experiment was to test if the algorithm would choose targets that are safe and can efficiently manage cluttered environments. Two experiments were carried out on this environment, Experiment I with a very high navigation cost and balanced parameter for exploration, and another one (Experiment II) with a lower 
navigation cost and a higher weight for the information gain parameter. Table VI shows the parameters used in both experiments.

Table VI

Parameters Used In The Experiments

\begin{tabular}{ccccc}
\hline & $\mathbf{W}_{\mathbf{A}}$ & $\mathbf{W}_{\mathbf{Q}}$ & $\mathbf{W}_{\mathbf{O}}$ & $\mathbf{W}_{\mathbf{C}}$ \\
\hline \hline Exp. I & 0.40 & 0.35 & 0.25 & 0.125 \\
Exp. II & 0.65 & 0.3 & 0.05 & 0.08 \\
\hline
\end{tabular}

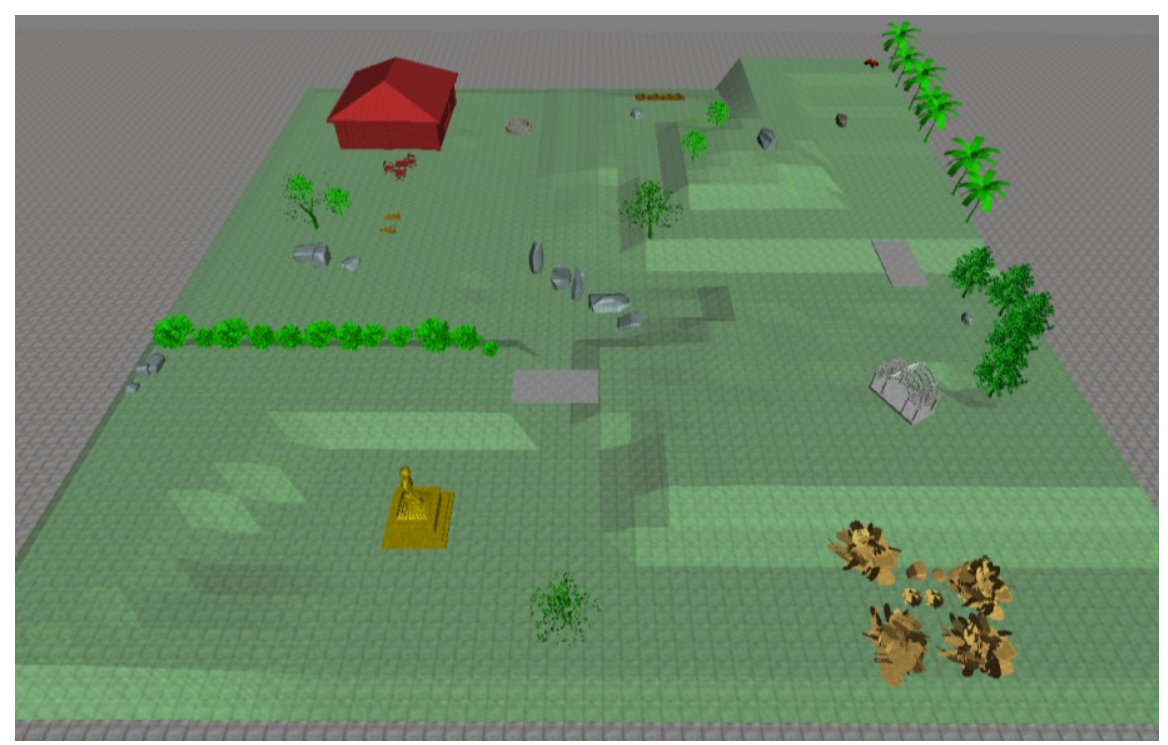

Figure 15 - Screenshot of the Simulated Cluttered Environment.

The resulting trajectories for both experiments are show in Figure 16.
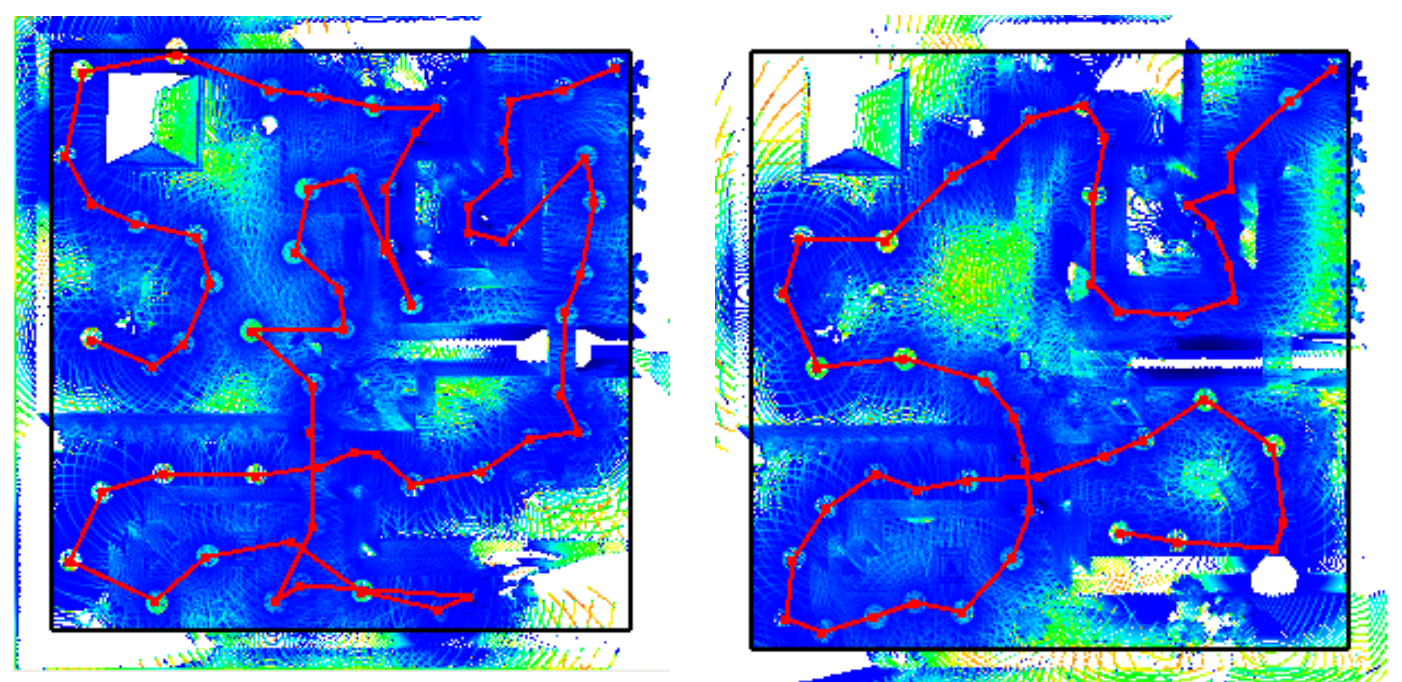

Figure 16 - Resulting Trajectories for Experiment I (left) and Experiment II (right). Colder Colours Represent Better Quality

Table VII shows the results of the experiments carried out in this environment, and Figure 17 shows the extracted navigation maps for both cases. 
Table VII

Results Obtained In Experiments

\begin{tabular}{ccccc}
\hline & $\begin{array}{c}\text { Travel } \\
\text { Distance }\end{array}$ & $\begin{array}{c}\text { Quality } \\
\text { Score }\end{array}$ & Coverage & Scans \\
\hline \hline Exp. I & $424 \mathrm{~m}$ & 0.7813 & $88 \%$ & 60 \\
Exp. II & $290 \mathrm{~m}$ & 0.6013 & $80 \%$ & 46 \\
\hline
\end{tabular}
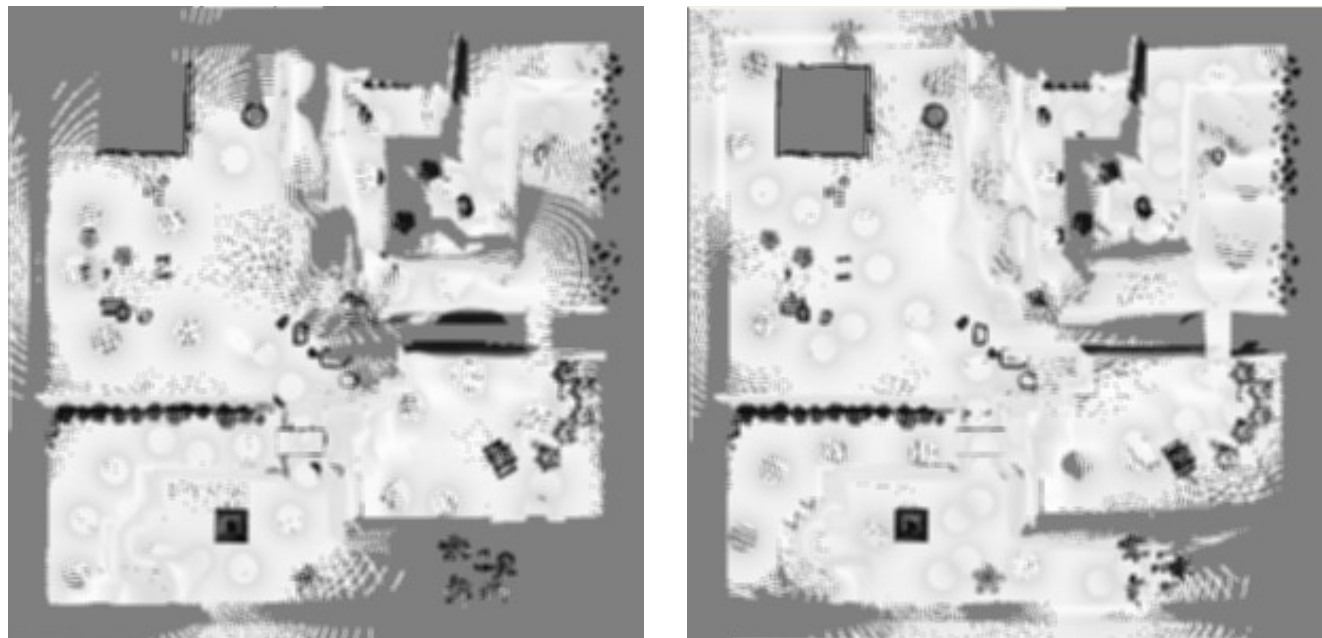

Figure 17 - Navigation maps; (left) Experiment I; (right) Experiment II.

In this case, results from both experiments are very different: experiment I made a much longer trajectory than experiment II and required $76 \%$ more scans; however, its quality score is much higher. On the other hand, experiment II covered almost as much area as experiment I using fewer scans and travelling half the distance. These differences are explained by two factors: first, navigation weight made the algorithm in the first experiment more conservative when choosing paths and second, the importance of quality improvement made it necessary to make more scans to cover the same area as in experiment II.

The area coverage difference can be explained by the interest zone coverage weight: in experiment II, when the house in the environment was scanned, the algorithm was driven away from it due to the need to scan uncovered zones. In experiment I, when the house was captured, many interest zones were created around the house and their importance made the system follow trajectories that covered the whole building. The reconstructed model can be seen in Figure 18 .

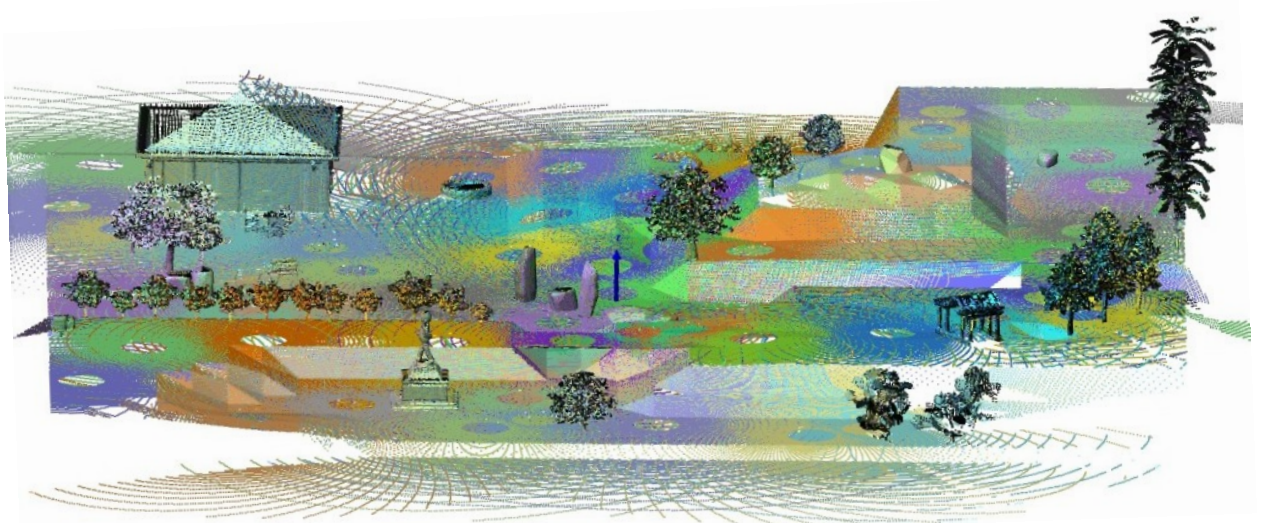

Figure 18 - Reconstructed model for experiment I. 


\subsection{Real Environments}

We have carried out experiments in two real environments. The first one is around the building of the CARTIF Foundation at Boecillo, Spain. This place was selected for several reasons, apart from being a very accessible place for our tests: it is a geometrically well defined building complex with access paths and grass all around which is useful to test our floor detection algorithm, and there are some obstacles and slopes suitable for testing the trajectory cost estimation system. Finally, even though it is geometrically simple, it still has several occlusion planes that are interesting to test the algorithm's performance on occlusions. The scanning site can be seen in Figure 19.

The second environment is an abandoned building called "El Pinaron" at Viana de Cega, Spain. This place was selected because it would be an interesting rescue scenario with a big structure surrounded by many trees and rubble, with different terrain heights and several occlusion planes. The scanning site can be seen in Figure 25.

\subsubsection{CARTIF Foundation Site}

Two different experiments have been carried out in this environment. The parameter values for the experiments were chosen to achieve different objectives. In the first experiment, the parameters were set to maximize the amount of information captured on each scan while, at the same time, reduce trajectory costs; in the second experiment, model quality was given more importance than the other parameters in order to obtain a good model of the site. However, the importance of trajectory cost was also increased. The selected values can be seen in Table VIII.

Table VIII

Parameters Used In The Experiments

\begin{tabular}{cccccc}
\hline & $\mathbf{w}_{\mathbf{A}}$ & $\mathbf{w}_{\mathbf{Q}}$ & $\mathbf{w}_{\mathbf{O}}$ & $\mathbf{w}_{\mathbf{C}}$ & Scans \\
\hline \hline Exp. I & 0.84 & 0.10 & 0.05 & 0.05 & 15 \\
Exp. II & 0.35 & 0.40 & 0.15 & 0.40 & 55 \\
\hline
\end{tabular}

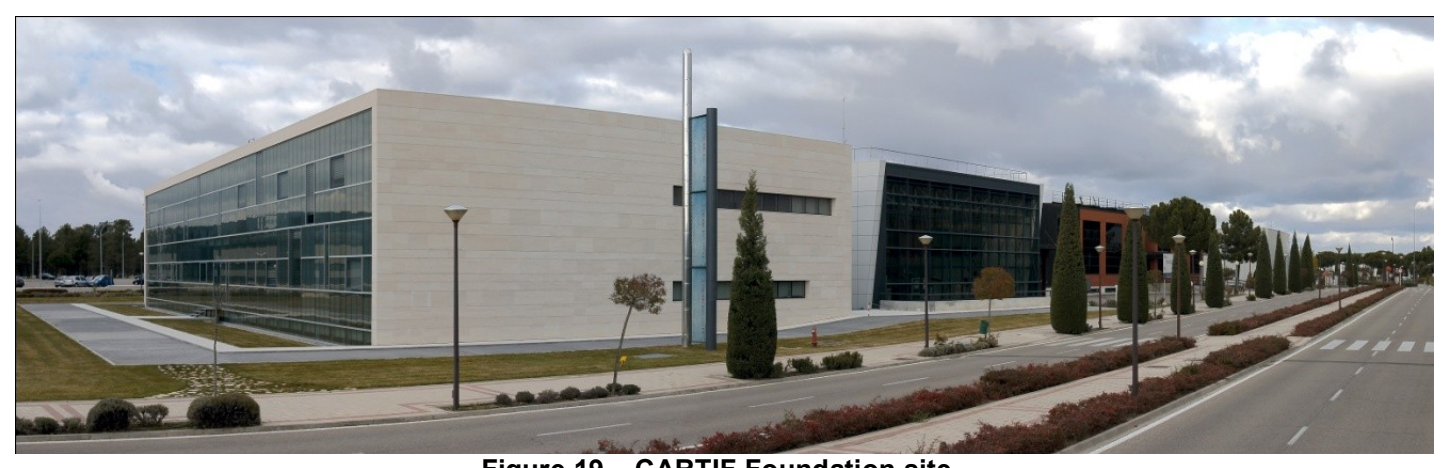

Figure 19 - CARTIF Foundation site.

\section{Extraction of safe navigation zones and obstacles}

The results obtained from this process in this scenario can be seen in Figure 20 and they show that our method can detect safe navigation zones and obstacles in real terrains with different 
heights and slopes. It can also be observed that obstacle detection is valid for objects at different heights and with different sizes.
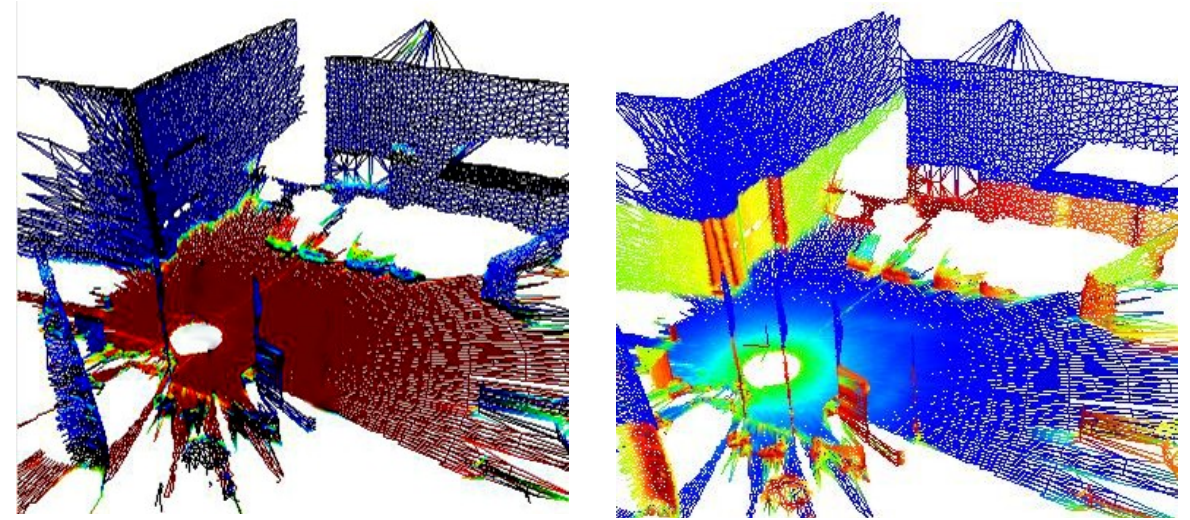

Figure 20 - Result of the ground (left) and obstacle (right) detection methods on a real scan. warmer colours represent detected surfaces

Using these data, a 2D navigation map was created (see Figure 21). The result is a good quality map, useful for path planning. It is also a proper plant map of the environment. When the proposed 2D navigation map is compared to typical occupancy grid maps, it is possible to see how the navigability concept is applied.

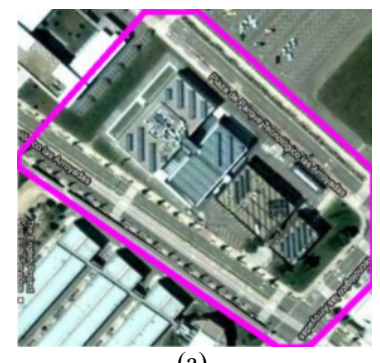

(a)

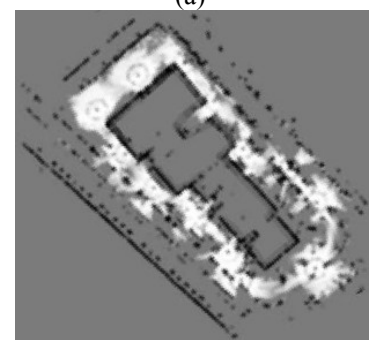

(b)

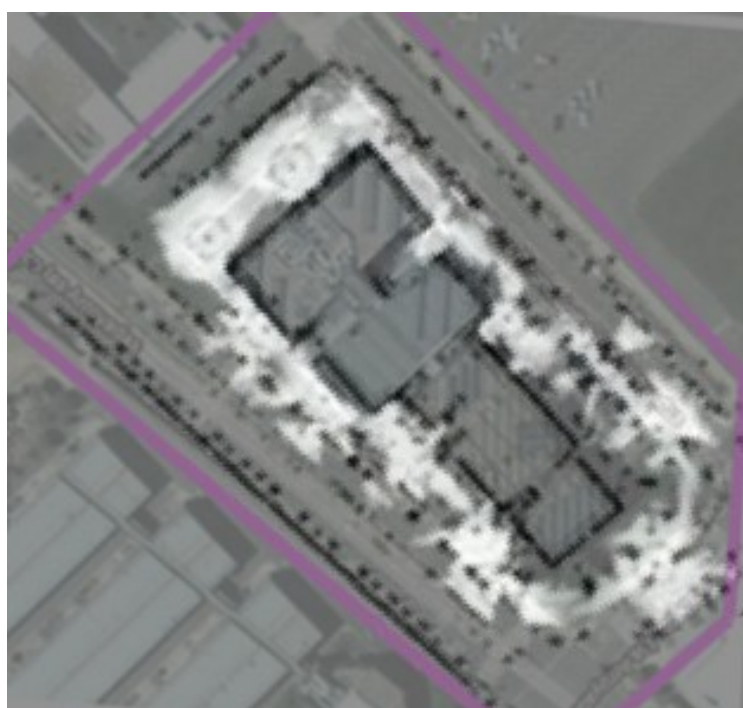

(c)

Figure 21 - (a) Aerial view of the CARTIF Foundation site. (b) resulting 2D navigation map. (c) Comparative image between the obtained map and the aerial view of the environment.

\section{Exploration Process Results}

Figure 22 shows the trajectory that the robot followed and the final model for experiment I. In the left image, colder colours represent higher quality scores and the magenta line shows the robot's trajectory. In the right image, each captured point cloud is represented by a different colour. 

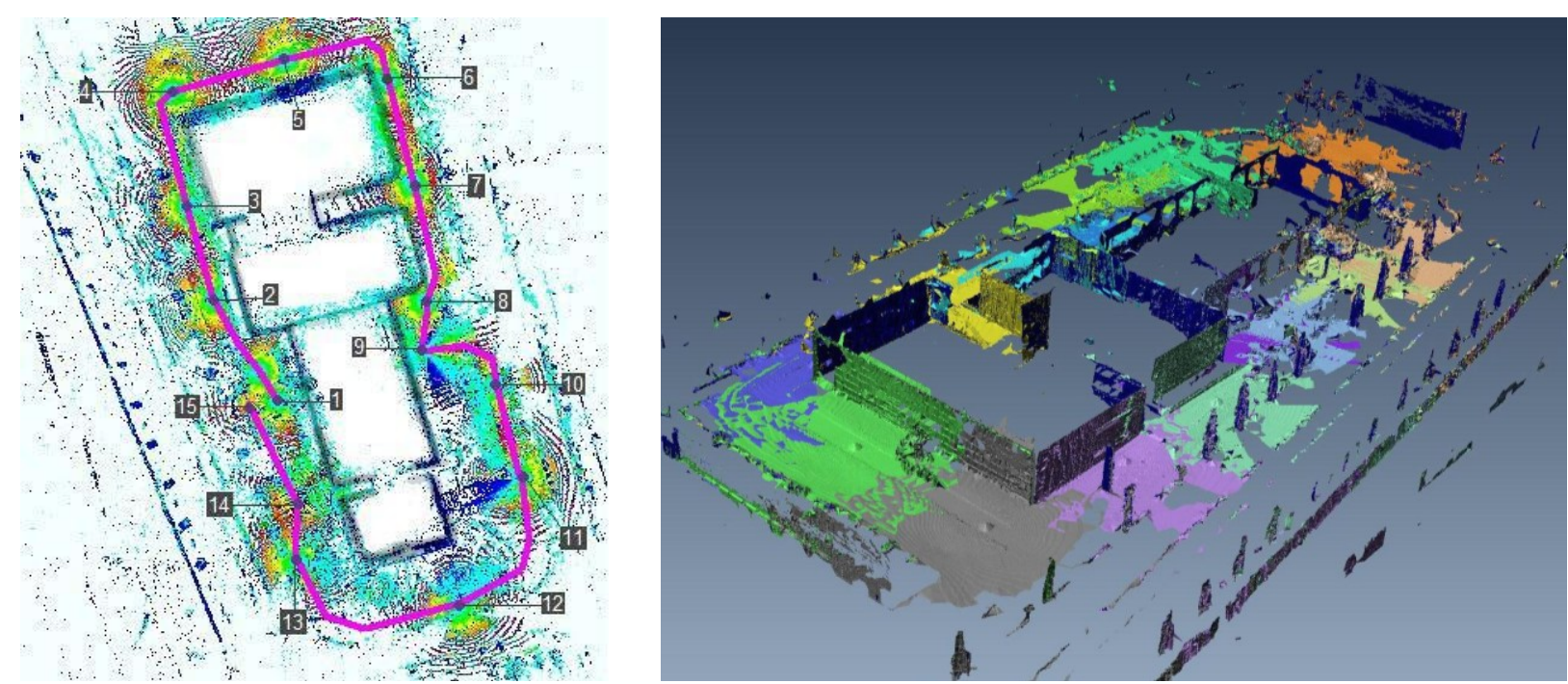

Figure 22 - Trajectory covered by the robot (in purple) in experiment I, where information gain was the most relevant criterion (left). Waypoints where scans were made are numbered in grey. Resulting model for the experiment (Right).
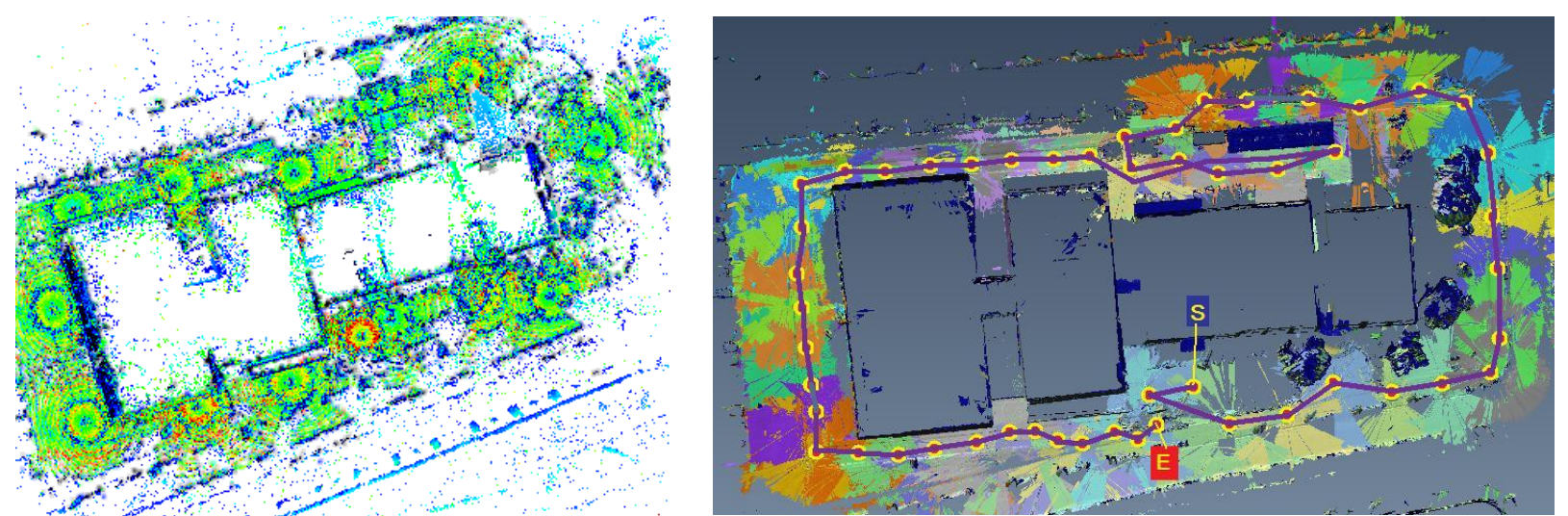

Figure 23 - Left. Resulting model quality score, colder colours represent higher quality. Right. Robot trajectory form point starting at point $S$.

Figure 23 shows the trajectory that the robot followed for experiment II. In the left image, colder colours represent higher quality scores, while in the right image, the magenta line shows the robot's trajectory. Figure 24, represents the final model after this test, where each captured point cloud is represented by a different colour.

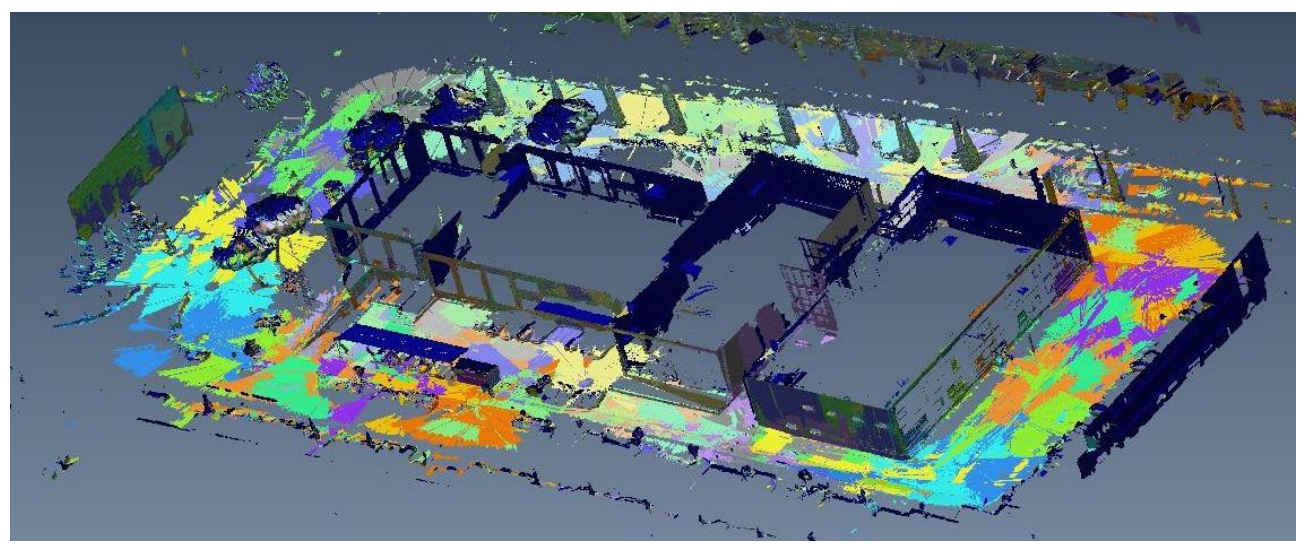

Figure 24 - Resulting model for experiment II 
In this case, both experiments provided very different results. The first and most relevant factor for this difference is that, in experiment II, quality was the dominating factor, which, along with an increment of the weight of the cost estimation factor, results in candidate targets close to the robot location to obtain a clear advantage over any other targets. However, in experiment I, quality was not really important compared to the information gain, which gave a clear offset to the score of the targets at the frontier between the known and unknown space. The second factor for the said difference is that the penalty that the robot turns had for the trajectory cost estimation factor, pushed the robot continuously towards the frontier of the known space, leaving unscanned candidate locations that would be interesting for the first experiment. However, the quality of the final model was acceptable. These results show that parameter tuning allows the system to behave in different ways, depending on the final concrete application of the system.

\subsection{2. "El Pinaron"}

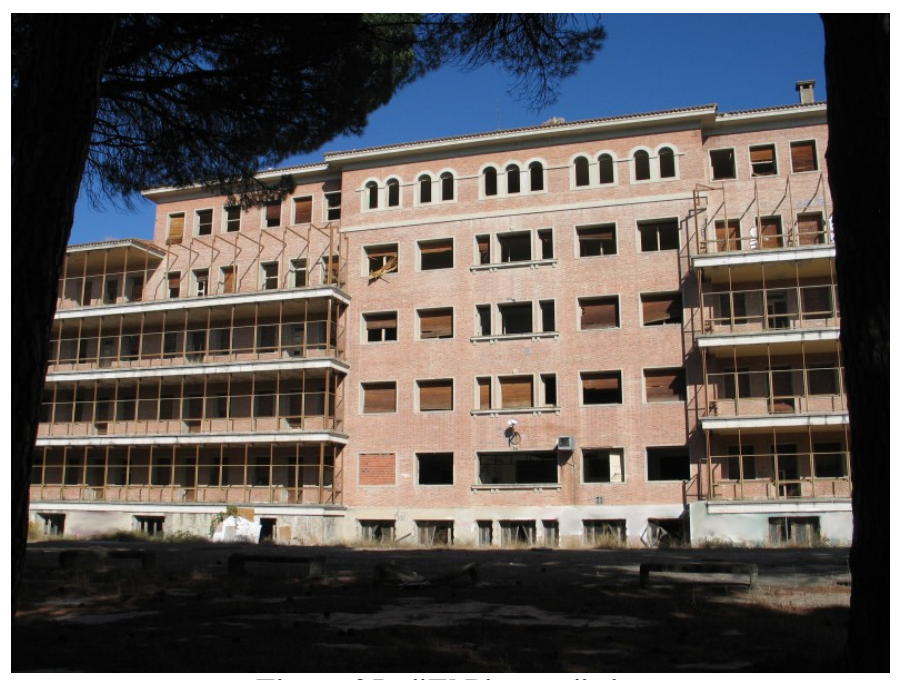

Figure 25 - "EI Pinaron" site

This site (see Figure 25) is a very interesting and challenging environment, since it has a big structure with long sides surrounded by trees and different obstacles, on a terrain with some slope and several occlusion planes. Moreover, it is an abandoned building, with through-time deteriorated structures access paths, which represents a perfect scenario for safety or rescue robotics.

In order to test how our system performs against typical stationary setups, we have designed an emergency simulacrum on which a collapsing building has to be quickly reconstructed to evaluate possible risks. For this test, we have used both our robot and a stationary system controlled by an experienced operator. First, we will show our system's performance on safe navigation zones extraction and exploration while reconstructing the environment. Then, we will comment on the performance of the stationary system, and finally, we will compare both scenarios by taking into account the comments made by the stationary system operator.

For the robotic exploration, we have set the parameters in a balanced way in order to maximize the amount of information captured on each scan while reducing trajectory costs. The selected parameter values can be seen in Table IX. 
Table IX

Parameters Used In The Experiment

\begin{tabular}{ccccc}
\hline & $\mathbf{w}_{\mathbf{A}}$ & $\mathbf{w}_{\mathbf{Q}}$ & $\mathbf{w}_{\mathbf{O}}$ & $\mathbf{w}_{\mathbf{C}}$ \\
\hline \hline Exp. I & 0.55 & 0.30 & 0.15 & 0.16 \\
\hline
\end{tabular}

\section{Extraction of safe navigation zones and obstacles}

The results obtained for this process in this scenario can be seen in Figure 26 and show that the proposed method can detect safe navigation zones and obstacles in irregular terrains.

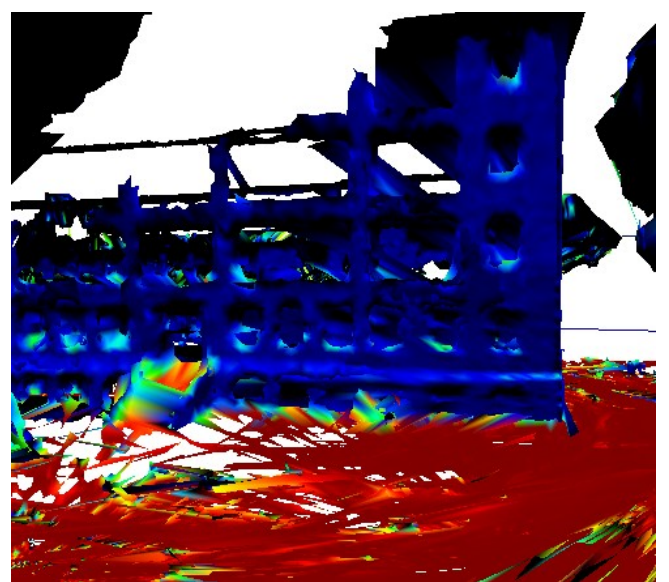

Figure 26 - Result of the ground detection method on a real scan. Warmer colours represent detected surfaces

The 2D navigation map created can be seen in Figure 27. The result is a good quality map, useful for path planning, and is also a proper plant map of the environment. When the proposed 2D navigation map is compared to typical occupancy grid maps, it is possible to see how the navigability concept is applied.

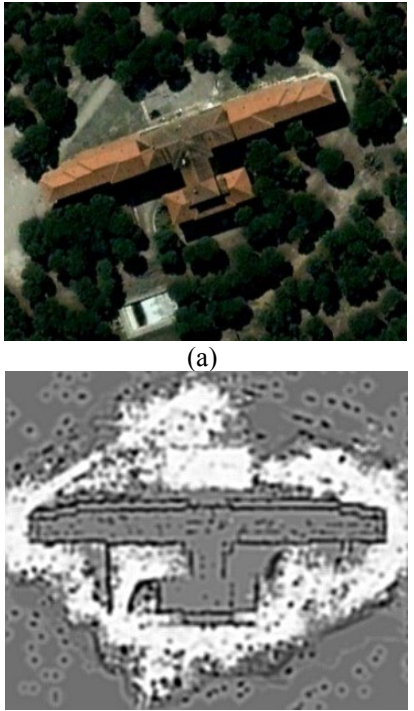

(b)

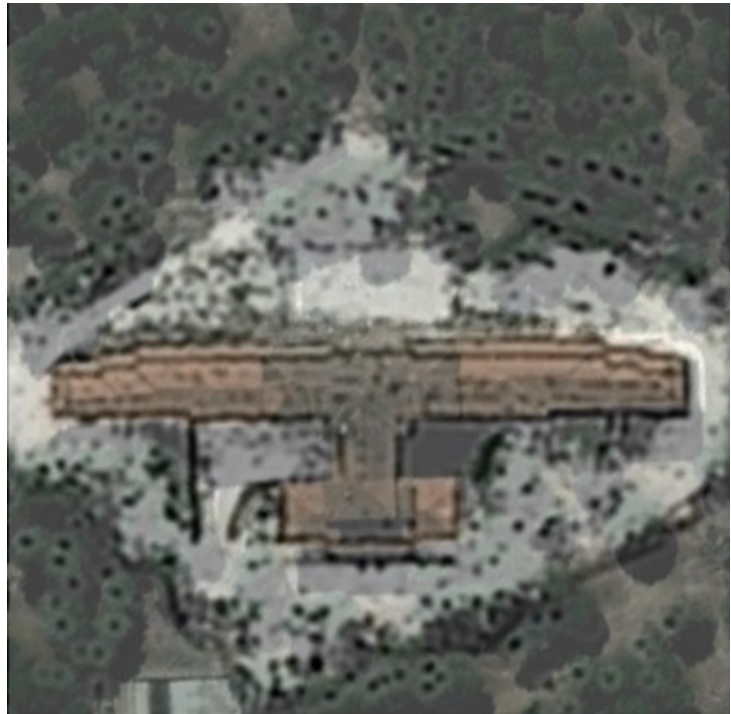

(c)

Figure 27 - (a) Aerial view of the scanning site. (b) resulting 2D navigation map. (c) Comparative image between the obtained 2D Navigation map and the aerial view of the environment 


\section{Exploration Process Results}

Figure 28 shows the trajectory that the robot followed and the final model for robotic exploration. In the image, colder colours represent higher quality scores and the magenta line shows the robot's trajectory.

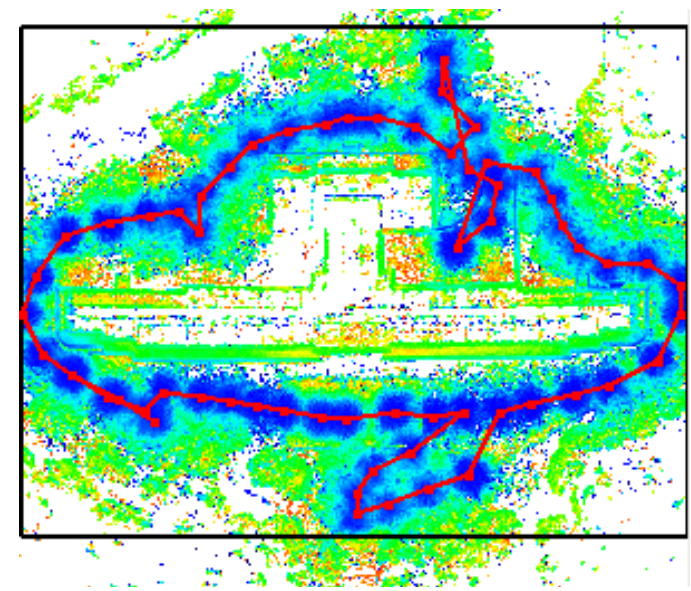

Figure 28 - Resulting Trajectory and model quality score, colder colours represent higher quality

The resulting trajectory shows that the robot can efficiently reconstruct a cluttered environment moving on rough terrain. The coverage for this environment was $62 \%$ of the explorable cells and the distance travelled was $690 \mathrm{~m}$. Some zones left uncovered were detected as untraversable because of high soil, and tree density as seen in the navigation map (Figure 27, right). Figure 29 shows the resulting model after the exploration process.
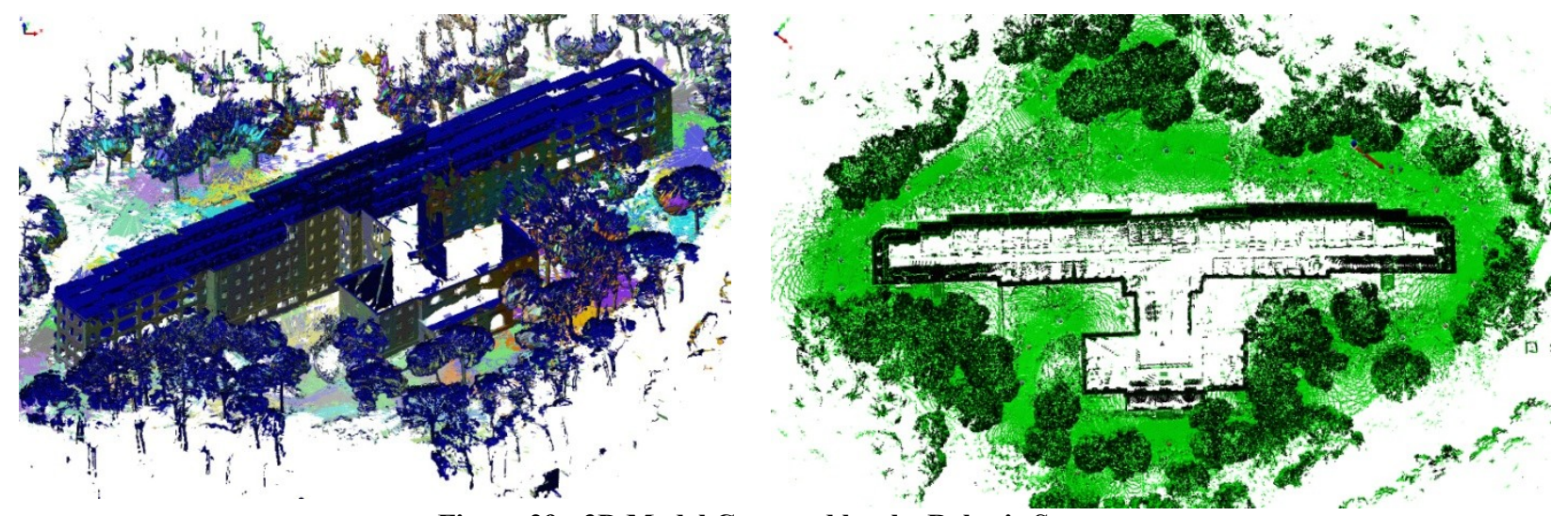

Figure 29 - 3D Model Captured by the Robotic System

\section{Stationary System Reconstruction}

For the stationary system, an expert operator using a LEICA HDS-3000 system made a partial reconstruction of the same environment, the results of which can be seen in Figure 30. The Leica system has a reach of $100 \mathrm{~m}$ and can reach resolutions of $1 \mathrm{~mm}^{2}$ from that distance, so the captured model had a quality that our system cannot reach. On the other hand, it needs a 5 minute setup time and, for this model, each scan required 15 minutes. Once every scan was captured, the system had to be shut down and manually taken to the new location where the whole process had to be repeated. The weight of the scanning system $(13 \mathrm{~kg})$ and its batteries $(8$ 
$\mathrm{kg}$ ), as well as the additional equipment (PC, tripod and GPS system) that had to be transported also make this hard for only one person, especially on unstructured cluttered environments where each piece of equipment has to be moved independently. Also, it has to be pointed out that in an SSRR scenario, where toxic gases, biological threats, radioactive elements or other substances may be present, this labour could be really dangerous for a human operator.

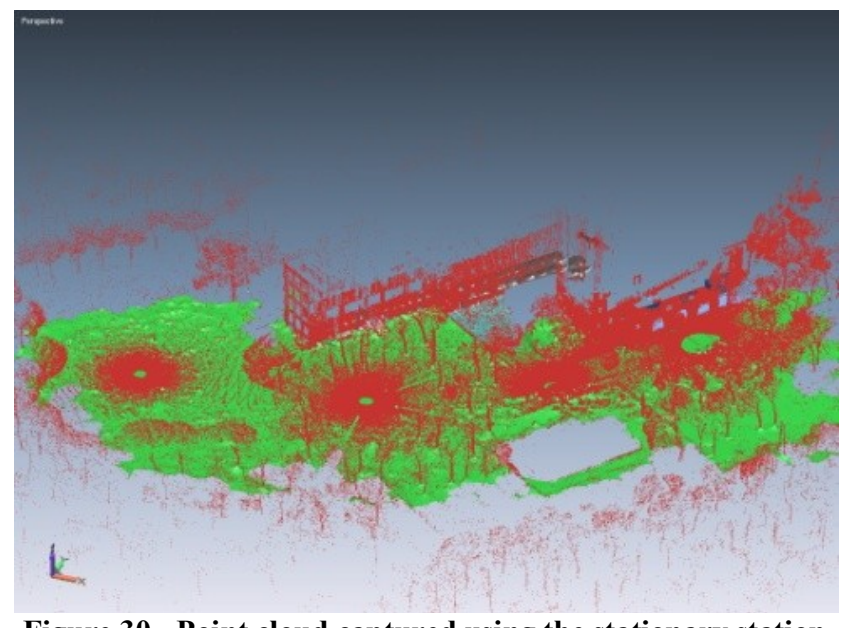

Figure 30 - Point cloud captured using the stationary station

The expert was also present whilst the robot reconstructed the same environment in order to make some comments on the reconstruction system. He pointed out that the stationary system would usually require very few scans to reconstruct one environment. However, for this case, in such a cluttered environment, it would require at least 20 scans due to the high number of occlusions, which, translated into time, would mean approximately 8 hours of labour, while our system did it in less than two hours.

\subsection{Execution Time Analysis}

In order to analyze algorithm execution times, the algorithm itself has to be split into different parts. First of all, the previous process times which include triangulation and 6D SLAM execution times, then the $3 \mathrm{D}$ analysis stage and finally the target evaluation stage, which is done for every target when deciding a new view.

Algorithm execution times vary greatly depending on the amount of $3 \mathrm{D}$ data to be processed. However, for these experiments, on a $3 \mathrm{GHz}$ Intel Core 2 Duo processor, the maximum processing times for each stage are shown in Table X.

Table X

Maximum Processing Times

\begin{tabular}{cc}
\multicolumn{2}{c}{ Maximum Processing Times } \\
\hline Process & $\begin{array}{c}\text { Maximum } \\
\text { Processing Time }\end{array}$ \\
\hline \hline Model Triangulation & $122 \mathrm{~ms}$ \\
6D SLAM & $8,492 \mathrm{~ms}$ \\
3D Analysis & $450 \mathrm{~ms}$ \\
Target Evaluation & $15,334 \mathrm{~ms}$ \\
\hline \hline Maximum Total Time & $24,398 \mathrm{~ms}$ \\
\hline
\end{tabular}


These results show that the algorithm can be executed online and that it does not delay the process much, especially considering that these are the worst cases for every process.

\section{Conclusion}

A method for the automatic reconstruction of outdoor environments has been presented. This method introduces an algorithm for efficiently planning viewpoints from available 3D data. Different criteria are used by this algorithm in order to obtain a model with a quality factor over a minimum desired value. The trajectory that the robot must follow to reach each possible target is also considered, so that the process keeps an accurate balance between the utility of a point and the cost of getting to it.

The way 3D data is processed in order to quantify the model quality and extract navigation surfaces, obstacles and interest regions has been discussed. A navigation map useful for 3D environments and its resemblance to $2 \mathrm{D}$ occupancy grid maps has also been introduced.

The obtained results show that our method can automatically reconstruct an outdoor 3D scene by calculating efficient trajectories for the robot, and that these trajectories change according to the chosen parameters to fulfill the desired criteria.

Our future work research will focus on optimizing the evaluation functions in order to make target evaluation parameters more lineal. We also believe that creating a learning system for training these parameters to imitate an expert user viewpoint selection could be a challenging research line.

Semantic labelling of objects from 3D clouds is also interesting and it could, in the future, help to develop new exploration techniques that consider many new different situations when evaluating the target. Finally, it may be interesting to work on an extension of this algorithm that can take into account other sensor readings that may be necessary for evaluating the environmental situation.

\section{Acknowledgement}

The authors would like to thank Mr. Jose Maria Llamas Fernandez for his help on evaluating our system against stationary laser scanning technology, and for many other useful recommendations. This work has been partially supported by the Spanish Comisión Interministerial de Ciencia y Tecnología, (CICYT) under Project DPI2008-06738-C01.

\section{REFERENCES}

Basilico, N., \& Amigoni, F. (2009). Exploration strategies based on multicriteria decision making for an autonomous mobile robot. Proceedings of ECMR, 2009, (pp. 259 - 264).

Blaer, P. S., \& Allen, P. K. (2007). Data Acquisition and View Planning for 3-D Modeling Tasks. IROS. San Diego, CA: IEEE/RSJ.

Blaer, P. S., \& Allen, P. K. (2009). View Planning and Automated Data Acquisition for ThreeDimensional Modeling of Complex Sites. Journal of Field Robotics , 26 (11-12), 865-891. 
Bourque, E., \& Dudek, G. (1998). Viewpoint selection-an autonomous robotic system for virtual environment creation. IROS (pp. 526-532). IEEE.

Danner, T., \& Kavraki, L. E. (2000). Randomized Planning for Short Inspection Paths. Proceedings of IEEE International Conference on Robotics and Automation ICRA.

Dellaert, F. (2005). 4D cities spatio-temporal reconstruction from images. Recuperado el 10 de 11 de 2010, de http://www.cc.gatech.edu/4d-cities/

Früh, C., \& Zakhor, A. (2003). Constructing 3D City Models by Merging Ground-Based and Airborne Views. IEEE Computer Society Conference on Computer Vision and Pattern Recognition (CVPR '03), (p. 562).

Gonzalez-Baños, H. H., \& Latombe, J. C. (2002). Navigation strategies for exploring indoor environments. International Journal of Robotics Research , 21, 829-848.

Grabowski, R., Khosla, P., \& Choset, H. (2003). Autonomous exploration via regions of interest. Proceedings of International Conference on Intelligent Robots and Systems (IROS 2003) (pp. 27-31). IEEE/RSJ.

Hertzberg, J., Lingemann, K., Christopher, L., Nüchter, A., \& Stiene, S. (2008). Does It Help a Robot Navigate to Call Navigability an Affordance? In Towards Affordance-Based Robot Control (pp. 16-26). Springer Berlin / Heidelberg.

Jensen, B., Weingarten, J., Kolski, S., \& Siegwart, R. (2005). Laser range imaging using mobile robots: From pose estimation to 3D-models. 1st Range Imaging Research Day, (pp. 129144). Zurich, Switzerland.

Newman, P., Bosse, M., \& Leonard, J. (2003). Autonomous feature-based exploration. Proceedings of the IEEE International Conference on Robotics and Automation, ICRA03 (pp. 1234-1240). IEEE.

Nüchter, A., Lingemann, K., \& Hertzberg, J. (2006). Extracting drivable surfaces in outdoor 6d slam. 37nd International Symposium on Robotics (ISR '06). Munich, Germany.

Nüchter, A., Lingemann, K., Hertzberg, J., \& Surmann, H. (2007). 6D SLAM - 3D mapping outdoor environments. Journal of Field Robotics , 24 (8-9), 699 - 722.

Ohno, K., Tadokoro, S., Nagatani, K., Koyanagi, E., \& Yoshida, T. (2009). 3-D mapping of an underground mall using a tracked vehicle with four sub-tracks. 2009 IEEE International Workshop on Safety, Security \& Rescue Robotics (SSRR), (pp. 1-6). Denver, CO.

Pathak, K., Birk, A., Vaskevicius, N., Pfingsthorn, M., Schwertfeger, S., \& Poppinga, J. (2010). Online three-dimensional SLAM by registration of large planar surface segments and closed-form pose-graph relaxation. Journal of Field Robotics , 27 (1), 52--84.

Pfaff, P., Triebel, R., Stachniss, C., Lamon, P., Burgard, W., \& Siegwart, R. (2007). Towards Mapping of Cities. IEEE International Conference on Robotics and Automation, (pp. 48074813). Roma.

Rusinkiewicz, S. (n.d.). Trimesh2. Retrieved 11 16, 2010, from http://www.cs.princeton.edu/gfx/proj/trimesh2/

Simmons, R. (2009, 11 9). Inter Process Communication (IPC). Retrieved 11 16, 2010, from http://www.cs.cmu.edu/ IPC/

Smith, R. (2007, May 28). Open Dynamics Engine (ODE). Retrieved 11 16, 2010, from http://www.ode.org/

Teller, S. (1997). Automatic acquisition of hierarchical, textured 3D geometric models of urban environments: Project Plan. Proceedings of the Image Understanding Workshop. New Orleans. 
Tovey, C., \& Koenig, S. (2003). Improved Analysis of Greedy Mapping. IEEE/RSJ International Conference on Intelligent Robots and Systems.

Williams, M. (2010). Street View: Explore the world at street level. Retrieved November 10, 2010, from Google Street View Web http://maps.google.es/intl/en_us/help/maps/streetview/

Yamauchi, B. (1997). A frontier-based approach for autonomous exploration. International Symposium on Computational Intelligence in Robotics and Automation, ICRA97, (pp. 146151).

Yamauchi, B., Schultz, A., Adams, W., \& Graves, K. (1998). Integrating map learning, localization and planning in a mobile robot. Intelligent Control (ISIC), (pp. 331-336). 